\title{
GEODESIC RIGIDITY IN COMPACT NONPOSITIVELY CURVED MANIFOLDS
}

\author{
BY \\ PATRICK EBERLEIN ${ }^{1}$
}

\begin{abstract}
Our goal is to find geometric properties that are shared by homotopically equivalent compact Riemannian manifolds of sectional curvature $K<0$. In this paper we consider mainly properties of free homotopy classes of closed curves. Each free homotopy class is represented by at least one smooth periodic geodesic, and the nonpositive curvature condition implies that any two periodic geodesic representatives are connected by a flat totally geodesic homotopy of periodic geodesic representatives. By imposing certain geometric conditions on these periodic geodesic representatives we define and study three types of free homotopy classes: Clifford, bounded and rank 1 . Let $M, M^{\prime}$ denote compact Riemannian manifolds with $K<0$, and let $\theta: \pi_{1}(M, m) \rightarrow \pi_{1}\left(M^{\prime}, m^{\prime}\right)$ be an isomorphism. Let $\theta$ also denote the induced bijection on free homotopy classes.

THEOREM A. The free homotopy class $[\alpha]$ in $M$ is, respectively, Clifford, bounded or rank 1 if and only if the class $\theta[\alpha]$ in $M^{\prime}$ is of the same type.

THEOREM B. If $M, M^{\prime}$ have dimension 3 and do not have a rank 1 free homotopy class then they have diffeomorphic finite covers of the form $S^{1} \times M^{2}$. The proofs of Theorems $\mathrm{A}$ and $\mathrm{B}$ use the fact that $\theta$ is induced by a homotopy equivalence $f$ : $(M, m) \rightarrow\left(M^{\prime}, m^{\prime}\right)$.

THEOREM C. The manifold $M$ satisfies the Visibility axiom if and only if $M^{\prime}$ satisfies the Visibility axiom.
\end{abstract}

Introduction. One form of the strong rigidity theorem of Mostow [22] states that if $M, M^{*}$ are compact, locally symmetric manifolds of nonpositive sectional curvature with no 1- or 2-dimensional local de Rham factors, then any isomorphism between the fundamental groups of $M$ and $M^{*}$ is induced by a diffeomorphism of $M$ onto $M^{*}$ which is an isometry if the metric of $M^{*}$ is multiplied by a suitable positive constant. Of course, if $M, M^{*}$ are allowed to be arbitrary compact manifolds of nonpositive sectional curvature, not necessarily locally symmetric, then one cannot expect that an isomorphism of the fundamental groups is induced by an isometry. In general, it is unknown if an isomorphism of the fundamental groups is induced by a diffeomorphism. If $M, M^{*}$ have dimension 2 , however, then a theorem of Fenchel and Nielsen asserts that any isomorphism of the fundamental groups of $M$ and $M^{*}$ is induced by a homeomorphism of $M$ onto $M^{*}$.

One does have available the following topological information. If $M, M^{*}$ are compact manifolds of nonpositive sectional curvature, then any isomorphism $\theta$ : $\pi_{1}(M, p) \rightarrow \pi_{1}\left(M^{*}, p^{*}\right)$ is induced by a continuous map $G:(M, p) \rightarrow\left(M^{*}, p^{*}\right)$. The

Received by the editors November 20, 1980.

1980 Mathematics Subject Classification. Primary 53C99, 53A35; Secondary 49F99.

Key words and phrases. Nonpositive curvature, free homotopy class, periodic geodesic, homotopy equivalence, Clifford, bounded, rank 1, Visibility axiom.

${ }^{1}$ Supported in part by NSF Grant MCS-7901730. 
map $G$ induces isomorphisms on all homotopy groups since $\pi_{k}$ is zero for $k>2$, and hence $G$ is a homotopy equivalence [25, p. 405]. Since homotopy equivalences induce isomorphisms on homology, it follows that compact, nonpositively curved manifolds $M, M^{*}$ with isomorphic fundamental groups have the same dimension and are both orientable or both nonorientable.

Given a homotopy equivalence between compact nonpositively curved manifolds $M, M^{*}$ one now has two obvious directions in which to proceed: (1) to look for conditions under which the manifolds are actually diffeomorphic; (2) to look for geometric properties that are preserved by homotopy equivalences. The first course has been followed in [15], [16] and the second in [4], [14], to mention a few examples. In this paper we follow the second course, and specifically we consider geometric properties of free homotopy classes of closed curves that are preserved by homotopy equivalences.

It is well known that any nontrivial free homotopy class of closed curves on a compact Riemannian manifold $X$ contains at least one smooth periodic geodesic. Our approach is to study free homotopy classes by studying the geometric properties of the smooth periodic geodesics that represent them. There may be more than one smooth periodic geodesic that is contained in a given free homotopy class $[\alpha]$, but if $X=M$ has nonpositive sectional curvature, then for any two smooth periodic geodesic representatives $\gamma_{0}, \gamma_{1}$ of $[\alpha]$ we can find a smooth, totally geodesic, isometric immersion $F: S^{1}(c) \times[0, R] \rightarrow M$ such that $F\left(S^{1} \times\{0\}\right)=\gamma_{0}$ and $F\left(S^{1} \times\{R\}\right)=\gamma_{1}$ (Proposition 3.1). In particular, $\gamma_{0}$ and $\gamma_{1}$ have the same length $c>0$ and can be joined by a family $\gamma_{t}=F\left(S^{1} \times\{t\}\right)$ of smooth periodic geodesics belonging to $[\alpha]$. The number $R=L_{F}\left(\gamma_{0}, \gamma_{1}\right)$ is the length of the homotopy $F$.

The flat homotopy $F$ is, in general, not unique, as we may see for flat tori. In fact Proposition 3.6 shows that $[\alpha]$ is bounded as defined below if and only if there are only finitely many flat homotopies joining any two given periodic geodesic representatives $\gamma_{0}, \gamma_{1}$ of $[\alpha]$.

In this paper we define and consider three types of free homotopy classes in a compact manifold $M$ of nonpositive sectional curvature. A free homotopy class $[\alpha]$ is bounded if there exists a number $R>0$ such that $L_{F}\left(\gamma_{0}, \gamma_{1}\right) \leqslant R$ for any two smooth periodic geodesics $\gamma_{0}, \gamma_{1}$ that represent $[\alpha]$ and any flat homotopy $F$ that joins $\gamma_{0}$ to $\gamma_{1}$. A class $[\alpha]$ is Clifford if for every point $p$ of $M$ there exists a smooth periodic geodesic that contains $p$ and belongs to $[\alpha]$. A class $[\alpha]$ has rank 1 if it contains a smooth periodic geodesic $\gamma$ of rank 1. (A geodesic $\gamma$ of $M$ has rank 1 if there exists no smooth, totally geodesic isometric immersion $F: \mathbf{R} \times[0, \infty) \rightarrow M$ with $F(t, 0)=\gamma(t)$ for all $t$.) Every Clifford class is unbounded and every rank 1 class is bounded. If $M$ admits a Clifford free homotopy class, then for any free homotopy class $[\alpha]$ there exists an integer $n \geqslant 1$ such that $[\alpha]^{n}$ is unbounded. In general there seem to be no other relationships between these three classes except in dimensions 2 and 3.

We now present our results. Let $M, M^{*}$ be compact, nonpositively curved manifolds, and let $\theta: \pi_{1}(M, p) \rightarrow \pi_{1}\left(M^{*}, p^{*}\right)$ be an isomorphism. (We remark that 
$\boldsymbol{\theta}$ induces a bijection of free homotopy classes since there is a one-one correspondence between free homotopy classes of closed curves and conjugacy classes of the fundamental group.) Our main result, Theorem A, states that a nontrivial free homotopy class $[\alpha]$ in $M$ is, respectively, Clifford, bounded or rank 1 if and only if the class $\theta[\alpha]$ in $M^{*}$ has the identical property. Theorem B states that if $M, M^{*}$ have dimension 3 and if one manifold admits no geodesic of rank 1 , then $M, M^{*}$ have finite coverings that are diffeomorphic. Theorem $\mathrm{C}$ states that if $M$ satisfies the Visibility axiom (defined in $\$ 1$ ), then $M^{*}$ also satisfies the Visibility axiom.

In the special case that $M$ and $M^{*}$ have the same underlying differentiable manifold $\bar{M}$, then Theorem A implies that (1) the property that a given free homotopy class be Clifford, bounded or rank 1 does not depend on the particular metric of nonpositive sectional curvature on $\bar{M} ;(2)$ in cases where two or more of the three classes above occur in $\bar{M}$ the automorphism group of the fundamental group of $\bar{M}$ has geometric constraints.

Clifford and bounded free homotopy classes seem to differ in a fundamental way from rank 1 free homotopy classes. Propositions 3.5 and 3.6 provide characterizations of the first two classes in terms of algebraic conditions on the corresponding conjugacy class of the fundamental group. These conditions are clearly preserved by isomorphisms, which immediately proves the assertions of Theorem $\mathbf{A}$ for bounded and Clifford free homotopy classes. The rank 1 property for free homotopy classes apparently does not have a similar easy algebraic formulation as the discussion and examples of $\$ 3$ show. To prove Theorem A for rank 1 classes we must apparently use a homotopy equivalence between $M$ and $M^{*}$ that is induced by the isomorphism of fundamental groups. Geometric properties of this homotopy equivalence are discussed in $\$ 2$, which is a slight variation of the discussion of pseudoisometries of [22]. The proof of Theorem A is presented in $\$ 4$. The key step in the proof of Theorem A for rank 1 free homotopy classes is Lemma A4, an elaboration of an idea of M. Morse [21] that has been used often, for example in [7], [14], [17] and [22].

Besides being the most difficult to study, the rank 1 free homotopy classes are also the most interesting and important of the three classes. W. Ballmann has shown in [1] that if $M$ is a compact nonpositively curved manifold that admits a rank 1 free homotopy class, then the geodesic flow in $T_{1} M$ is topologically mixing; that is, given open sets $O, U$ of $T_{1} M$ there exists a number $A>0$ such that $T_{t}(O) \cap U$ is nonempty for $|t| \geqslant A$, where $\left\{T_{t}\right\}$ denotes the geodesic flow. Moreover, most of the strong properties of Visibility manifolds are shared by the much larger containing class of rank 1 manifolds, those admitting a rank 1 free homotopy class. If $M, M^{*}$ are compact nonpositively curved manifolds with isomorphic fundamental groups, then by Theorem A the existence of a rank 1 free homotopy class in $M$ implies the existence of a rank 1 free homotopy class in $M^{*}$, and hence the fundamental group isomorphism carries along with it the topological mixing property of the geodesic flow. We conjecture that if the geodesic flow is topologically transitive in $T_{1} M$ (has a dense orbit), then $M$ admits a rank 1 free homotopy class. If this conjecture were true, it would then follow that topological mixing and 
topological transitivity of the geodesic flow in $T_{1} M$ are equivalent properties (although the former appears to be much stronger) and that both are preserved by fundamental group isomorphisms or equivalently by homotopy equivalences.

If $M$ is a Visibility manifold, then every free homotopy class of $M$ is bounded, a property that by Proposition 3.6 is equivalent to the Preissmann condition that every abelian subgroup of $\pi_{1}(M)$ be infinite cyclic. It is unknown if the Preissmann condition implies the Visibility axiom in dimensions $>3$. Having no convenient formulation (yet) of the Visibility axiom in terms of properties of free homotopy classes, the proof of Theorem $\mathrm{C}$ must take a somewhat different course. Using a homotopy equivalence $G:(M, p) \rightarrow\left(M^{*}, p^{*}\right)$ induced by the isomorphism $\theta$ : $\pi_{1}(M, p) \rightarrow \pi_{1}\left(M^{*}, p^{*}\right)$, one can prove a geometric comparison result $(\S 5)$ that generalizes slightly Proposition 5.5 of [7]. (This result in turn is a generalization of the same idea of Morse [21] that we mentioned earlier.) Given the geodesic comparison result the proof of Theorem $\mathrm{C}$ now proceeds exactly as in the proof of Theorem 5.1 of [7].

Although Theorem B clearly fits into the general program described in the first paragraph of this introduction, its proof does not use a homotopy equivalence between $M$ and $M^{*}$ as do the proofs of Theorems $A$ and C. Instead the results rely heavily on the work of $\$ 5$ of [1].

We conclude the introduction with a description of the organization of the paper. The first section summarizes some basic facts about manifolds of nonpositive sectional curvature. In the second section we give a brief description of the construction of a continuous map $G:(M, p) \rightarrow\left(M^{*}, p^{*}\right)$ that realizes a given homomorphism $\theta: \pi_{1}(M, p) \rightarrow \pi_{1}\left(M^{*}, p^{*}\right)$. In the third section we discuss free homotopy classes of closed curves on a compact Riemannian manifold. We begin the section with some general facts about free homotopy classes that apply to any compact Riemannian manifold. We conclude the section with a discussion and comparison of Clifford, bounded and rank 1 free homotopy classes in a compact manifold $M$ with nonpositive sectional curvature. In $\$ 4$ we state and prove Theorems A and B. The proof of Theorem A for rank 1 free homotopy classes involves several technical lemmas which are only stated in $\$ 4$ and proved in Appendix III. Following the statement of the lemmas, a short argument concludes the proof of Theorem A. In $\$ 5$ we prove Theorem C.

In $\S \S 2$ and 3 we have often omitted entirely the proofs of certain results to clarify the exposition. All nontrivial missing proofs from these sections may be found in Appendices I and II. For the same reason we have omitted the proof of the technical lemmas in $\$ 4$ and have placed them in Appendix III.

I am indebted to $R$. Bishop for pointing out an error in the definition of a bounded free homotopy class that was used in an earlier version of this paper. He has also provided a way to view the differences between rank 1 and bounded free homotopy classes. See the discussion following Proposition 3.7.

1. Preliminaries (general reference [11]). We use the notation and basic results of [11], some of which we sketch here. Let $H$ denote a complete, simply connected Riemannian manifold of nonpositive sectional curvature. $H$ is sometimes called a 
Hadamard manifold. Let $d($, ) denote the Riemannian metric and $\langle$,$\rangle the inner$ product on the tangent spaces. All geodesics in $H$ or any quotient manifold $H / \Gamma$ are assumed to have unit speed. Unit speed geodesics $\gamma, \sigma$ of $H$ are asymptotes if there exists a number $c>0$ such that $d(\gamma t, \sigma t) \leqslant c$ for all $t \geqslant 0$. An equivalence class of asymptotic geodesics defines a point at infinity for $H$. For every geodesic $\gamma$ of $H$ let $\gamma(\infty)$ and $\gamma(-\infty)$ denote the points at infinity determined by $\gamma$ and $\gamma^{-1}$ : $t \rightarrow \gamma(-t)$, respectively. If $H(\infty)$ denotes the set of all points at infinity for $H$, then $\bar{H}=H \cup H(\infty)$ with a natural topology is homeomorphic to the closed unit $n$-ball in $\mathbf{R}^{n}, n=$ dimension $H$, and contains $H$ as a dense open subset. Isometries of $H$ extend to homeomorphisms of $\bar{H}$ : given an isometry $\phi$ of $H$ and a point $x \in$ $H(\infty)$, we define $\phi(x)=(\phi \circ \gamma)(\infty)$, where $\gamma$ is any geodesic belonging to $x$.

If $p, q$ are distinct points of $H$, then there exists a unique geodesic $\gamma_{p q}$ such that $\gamma_{p q}(0)=p$ and $\gamma_{p q}(a)=q$, where $a=d(p, q)$. If $p \in H$ and $x \in H(\infty)$ are any points, then there exists a unique geodesic $\gamma_{p x}$ of $H$ such that $\gamma_{p x}(0)=p$ and $\gamma_{p x}(\infty)=x$. The uniqueness of a geodesic between any two fixed points implies that $H$ is diffeomorphic to a Euclidean space, and hence we may define angle measurement as in a Euclidean space: given distinct points $p, q, r$ of $\bar{H}$ such that $p \in H$ we define $\Varangle_{p}(q, r)$ to be the angle subtended at $p$ by the vectors $\gamma_{p q}^{\prime}(0)$ and $\gamma_{p r}^{\prime}(0)$. The fact that $H$ is diffeomorphic to Euclidean space also implies that the homotopy groups $\pi_{k}(M), k \geqslant 2$, are zero for any quotient manifold $M=H / \Gamma$. Hence all the homotopy of a nonpositively curved manifold is concentrated in the fundamental group.

Axioms. $H$ is said to satisfy the Visibility axiom if for each point $p \in H$ and each number $\varepsilon>0$ there exists a constant $R=R(p, \varepsilon)>0$ such that $\Varangle_{p}(\sigma a, \sigma b)<\varepsilon$ if $\sigma:[a, b] \rightarrow H$ is any geodesic segment with $d(p, \sigma) \geqslant R$. One can also show that $H$ satisfies the Visibility axiom if and only if for any distinct points $x, y$ in $H(\infty)$ there exists a geodesic $\gamma$ of $H$ with $\gamma(\infty)=x$ and $\gamma(-\infty)=y . H$ or any quotient manifold $H / \Gamma$ is a Visibility manifold if $H$ satisfies the Visibility axiom. Visibility manifolds include all manifolds with sectional curvature $K \leqslant c<0$.

A flat strip, rectangle, half-plane or plane in a manifold $M$ of nonpositive sectional curvature is the image of a smooth, totally geodesic, isometric immersion $F: D \rightarrow M$, where $D$ is, respectively, $\mathbf{R} \times[0, a],[0, a] \times[0, b], \mathbf{R} \times[0, \infty)$ or $\mathbf{R}^{2}$. The geodesics of $M$ in $F(\partial D)$ will be called the boundaries of $F(D)$. If $M=H$, a simply connected manifold, then $F$ is necessarily an imbedding since any two points of $H$ are joined by a unique geodesic.

We say that a maximal geodesic $\gamma$ of $M$ (or $H$ ) has rank 1 if it is not the boundary of a flat half-plane in $M$ (or $H$ ), and a manifold $M$ (or $H$ ) has rank 1 if it admits a geodesic of rank 1 . If $H$ satisfies the Visibility axiom, then $H$ has rank 1, but the converse is false. If there exists a compact set $C \subseteq H$ such that $H$ is the union of the images of $C$ under isometries of $H$, then we have the following: $H$ satisfies the Visibility axiom if and only if $H$ contains no flat plane [7, Theorem 4.1].

Two maximal geodesics $\gamma, \sigma$ of $H$ are equivalent if $\gamma(\infty)=\sigma(\infty)$ and $\gamma(-\infty)=$ $\sigma(-\infty)$ for suitable orientations of $\gamma$ and $\sigma$. By Proposition 5.1 of [11] it follows 
that $\gamma$ and $\sigma$ are equivalent in $H$ if and only if $\gamma$ and $\sigma$ are the boundaries of a flat strip in $H$, and in this case $d(\gamma t, \sigma)$ and $d(\sigma t, \gamma)$ are the same constant function on R. From this it follows that if $\gamma$ and $\sigma$ are equivalent geodesics in $H$, then $\sigma$ has rank 1 if $\gamma$ has rank 1 . If $\sigma$ did not have rank 1 , then we could find a flat half-plane $F$ with boundary $\sigma$ and a sequence $\left\{\sigma_{n}\right\}$ of maximal geodesics contained in $F$ and equivalent to $\sigma$ such that $d\left(\sigma t, \sigma_{n}\right) \equiv C_{n} \rightarrow+\infty$ as $n \rightarrow+\infty$. It would follow that $\gamma$ and $\sigma_{n}$ are equivalent geodesics with $d\left(\gamma t, \sigma_{n}\right) \equiv b_{n} \rightarrow+\infty$. If $G_{n}$ is the flat strip of width $b_{n}$ bounded by $\gamma$ and $\sigma_{n}$, then $G_{n}$ would converge to a flat half-plane with boundary $\gamma$, contradicting the hypothesis that $\gamma$ has rank 1 .

Isometries. Let $I(H)$ denote the group of isometries of $H . I(H)$ is a Lie group with the compact open topology. To each isometry $\phi$ we associate a displacement function $d_{\phi}: p \rightarrow d(p, \phi p)$. The function $\left(d_{\phi}\right)^{2}$ is a $C^{\infty}$ convex function on $H$ by Proposition 4.2 of [2]. We say that an isometry $\phi$ is elliptic, hyperbolic or parabolic if $d_{\phi}$ has, respectively, zero minimum, positive minimum or no minimum in $H$. If $\phi$ is hyperbolic and $\left(d_{\phi}\right)^{2}$ has a positive minimum at $p \in H$, then $\phi$ translates the geodesic $\gamma=\gamma_{p \phi p}$; that is, $(\phi \circ \gamma)(t)=\gamma(t+\omega)$ for all $t \in \mathbf{R}$ and some $\omega>0$, the period of $\phi$. If $C_{\phi}$ denotes the minimum set of $\left(d_{\phi}\right)^{2}$, then $C_{\phi}$ is the union of all geodesics translated by $\phi$. For further details see Proposition 4.2 of [2].

The symbol $\Gamma$ will always denote a freely acting, properly discontinuous group of isometries of $H$, a condition equivalent to requiring that the quotient space $H / \Gamma$ be smooth. If $H / \Gamma$ has finite volume then $\Gamma$ is a lattice in $H$ and is uniform or nonuniform according to whether $H / \Gamma$ is compact or noncompact. A lattice $\Gamma$ is reducible if there exists a subgroup $\Gamma^{*}$ of finite index in $\Gamma$ such that $M^{*}=H / \Gamma^{*}$ is a Riemannian product $M_{1}^{*} \times M_{2}^{*}$ of manifolds of positive dimension.

Clifford translations and de Rham decompositions [18], [27]. Every Hadamard manifold $H$ is isometric to a Riemannian product $H_{0} \times H_{1} \times \cdots \times H_{k}$, where $H_{0}$ is a Euclidean space and $H_{i}$ is irreducible (cannot be expressed as a nontrivial Riemannian product) for every $i \geqslant 1$. This de Rham decomposition is unique up to the order of the factors $H_{i}$. An isometry $\phi$ of $H$ permutes the foliations $N_{i}$ tangent to the factors $H_{i}$. An isometry $\phi$ of an arbitrary space $H$ is a Clifford translation if the displacement function $d_{\phi}$ is constant in $H$. If $I(H)$ contains nonidentity Clifford translations, then $H$ admits a nontrivial Euclidean de Rham factor $H_{0}$ upon which the Clifford translations act as Euclidean translations while acting by the identity on the product of all non-Euclidean de Rham factors [27].

2. Induced maps and pseudoisometries. Let there be given two compact nonpositively curved manifolds $M=H / \Gamma$ and $M^{*}=H^{*} / \Gamma^{*}$ and a homomorphism $\theta$ : $\pi_{1}(M, p) \rightarrow \pi_{1}\left(M^{*}, p^{*}\right)$. It is known and elementary that there exists a continuous map $G:(M, p) \rightarrow\left(M^{*}, p^{*}\right)$ that induces the homomorphism $\theta$. For completeness we sketch a proof below. If $\phi$ is an isomorphism and if $\tilde{G}: H \rightarrow H^{*}$ is a lift of $G$, then $\tilde{G}$ is a $(\zeta, r)$ pseudoisometry for some positive constants $\zeta, r$. Definitions and precise statements are given below.

Proposition 2.1. Let $M, M^{*}$ be compact manifolds of nonpositive sectional curvature, and let $\theta: \pi_{1}(M, p) \rightarrow \pi_{1}\left(M^{*}, p^{*}\right)$ be a homomorphism. Then there exists a continuous map $G:(M, p) \rightarrow\left(M^{*}, p^{*}\right)$ such that $G$ induces the homomorphism $\theta$. 
REMARK. If $M, M^{*}$ have dimension 2 and $\theta$ is an isomorphism, then $G$ may be chosen to be a homeomorphism by a theorem of Fenchel and Nielsen [12], [20]. In general the map $G$ is only a homotopy equivalence.

Proof. This is in the spirit of [26, pp. 148-151]. Triangulate $M$ so that $p$ is one of the vertices of the triangulation and so that each simplex of maximal dimension lies in an open set of $M$ that is evenly covered by the projection $\pi: H \rightarrow M=H / \Gamma$. By means of $\pi$ we may lift this triangulation to obtain a $\Gamma$-invariant triangulation of $H$. Next, extend $\theta$ to a homomorphism from $\Gamma$ into $\Gamma^{*}$, the deckgroup of $M^{*}$, as follows. Choose $\tilde{p} \in \pi^{-1}(p)$ and $\tilde{p}^{*} \in\left(\pi^{*}\right)^{-1}\left(p^{*}\right)$, where $\pi^{*}: H^{*} \rightarrow M^{*}=H^{*} / \Gamma^{*}$ is the projection. Then for every $\gamma \in \Gamma$ let $\theta(\gamma)$ be that element $\gamma^{*}$ in $\Gamma^{*}$ such that $\theta$ maps the homotopy class of $\pi \circ \alpha$ into the homotopy class of $\pi^{*} \circ \alpha^{*}$, where $\alpha$ is the geodesic segment from $\tilde{p}$ to $\gamma \tilde{p}$ and $\alpha^{*}$ is the geodesic segment from $\tilde{p}^{*}$ to $\gamma^{*} \tilde{p}^{*}$. It is easy to check that $\theta$ is a homomorphism and well defined up to composition with an inner automorphism of $\Gamma^{*}$.

Let $\Sigma_{m}$ denote the $m$-skeleton of the $\Gamma$-invariant triangulation of $H$. We construct inductively over the sets $\Sigma_{m}$ a map $\tilde{G}: H \rightarrow H^{*}$ such that $\tilde{G}(\phi q)=\theta(\phi) \tilde{G}(q)$ for every $q \in H$ and every $\phi \in \Gamma$. $\tilde{G}$ will then induce the desired map $G$ : $(M, p) \rightarrow\left(M^{*}, p^{*}\right)$. To start we choose points $\left\{p_{1}, \ldots, p_{k}\right\}$ in $\Sigma_{0}$ such that for each point $q \in \Sigma_{0}$ there exists a unique $\phi \in \Gamma$ such that $\phi q \in\left\{p_{1}, \ldots, p_{k}\right\}$. We also assume that $\tilde{p}$ is one of the points $\left\{p_{i}\right\}$, where $\tilde{p} \in \pi^{-1}(p)$ is the point of the previous paragraph. Define $\tilde{G}(\tilde{p})=\tilde{p}^{*}$ and assign the other values $\tilde{G}\left(p_{i}\right), 1<i<$ $k$, arbitrarily in $H^{*}$. Finally extend $\tilde{G}$ over $\Sigma_{0}$ so that $\tilde{G} \circ \phi=\theta(\phi) \circ \tilde{G}$ in $\Sigma_{0}$. Next, suppose that we have constructed a map $\tilde{G}: \Sigma_{m} \rightarrow H^{*}$ such that $\tilde{G} \circ \phi=\theta(\phi) \circ \tilde{G}$ for $\phi \in \Gamma$. To extend $\tilde{G} \theta$-equivariantly to $\Sigma_{m+1}$ we choose $(m+1)$-simplices $\left\{\sigma_{1}, \ldots, \sigma_{t}\right\}$ such that for any simplex $\sigma \in \Sigma_{m+1}$ there exists a unique $\phi \in \Gamma$ such that $\phi(\sigma) \in\left\{\sigma_{1}, \ldots, \sigma_{t}\right\}$. Now $\tilde{G}$ is defined on $\partial \sigma_{i}$ for each $1<i<t$ and, since $H^{*}$ is contractible, we may extend $\tilde{G}$ continuously over each $\sigma_{i}$. We then define $\tilde{G}$ on $\phi\left(\sigma_{i}\right)$ by setting $\tilde{G}(\phi p)=\theta(\phi) \tilde{G}(p)$ for each point $p \in \sigma_{i}$.

After a finite number of steps we obtain a continuous map $\tilde{G}: H \rightarrow H^{*}$ such that $\tilde{G}(\phi q)=\theta(\phi) \tilde{G}(q)$ for every $q \in H$ and every $\phi \in \Gamma$. We then define $G: M \rightarrow M^{*}$ by $G \circ \pi=\pi^{*} \circ \tilde{G}$. The details of the construction show that $G$ is well defined, $G(p)=p^{*}$ and $G$ induces the homomorphism $\theta: \pi_{1}(M, p) \rightarrow \pi_{1}\left(M^{*}, p^{*}\right)$.

Definition. A continuous function $\tilde{G}: H \rightarrow H^{*}$ between two Hadamard manifolds will be called a $(\zeta, r)$ pseudoisometry relative to positive constants $\zeta, r$ if $\tilde{G}$ is uniformly continuous and if $d(\tilde{G} p, \tilde{G} q) \geqslant \zeta d(p, q)$ whenever $d(p, q)>r$.

Our definition is somewhat weaker than the definition of $[22$, p. 66], but it is sufficient for our purposes here and will be more convenient in [8]. We remark that the uniform continuity of $\tilde{G}$ implies the following two elementary facts:

(1) for every $\delta>0$ there exists a number $K>0$ such that $d(\tilde{G} p, \tilde{G} q) \leqslant K d(p, q)$ if $d(p, q) \geqslant \delta$.

(2) For every constant $r>0$ there exists a constant $B>0$ such that $d(\tilde{G} p, \tilde{G} q)$ $\leqslant B$ if $d(p, q) \leqslant r$.

Our next result is a key ingredient for the proof of Theorem $A$ in $\$ 4$. Its proof may be found in Appendix I. For a similar result see [22, pp. 66-70]. 
Proposition 2.2. Let $M=H / \Gamma$ and $M^{*}=H^{*} / \Gamma^{*}$ be compact manifolds of nonpositive sectional curvature, and let $\pi: H \rightarrow M$ and $\pi^{*}: H^{*} \rightarrow M^{*}$ denote the projections. Let $G:(M, p) \rightarrow\left(M^{*}, p^{*}\right)$ be a continuous map that induces an isomorphism $\theta: \pi_{1}(M, p) \rightarrow \pi_{1}\left(M^{*}, p^{*}\right)$, and let $\tilde{G}: H \rightarrow H^{*}$ be any lift of $G\left(\pi^{*} \circ \tilde{G}=\right.$ $G \circ \pi)$. Then $\tilde{G}$ is a $(\zeta, r)$ pseudoisometry for suitable positive constants $\zeta, r$, and $\tilde{G}(\phi q)=\theta(\phi) \tilde{G}(q)$ for all $q \in H$ and all $\phi \in \Gamma$, where the isomorphism $\theta: \Gamma \rightarrow \Gamma^{*}$ is induced by $G$.

3. Free homotopy classes. For the rest of this paper $M, M^{*}$ will denote compact manifolds of nonpositive sectional curvature. In this section, we first define free homotopy classes for an arc connected topological space $X$ and list some general facts. For compact nonpositively curved manifolds $M$ we define and study the relationships between three types of free homotopy classes: Clifford, bounded and rank 1 classes. For clarity the proofs of all assertions are omitted and the nontrivial proofs may be found in Appendix II.

Let $X$ be an arc connected topological space. Two continuous closed curves $\alpha$ : $[0,1] \rightarrow X$ and $\beta:[0,1] \rightarrow X$ are said to be freely homotopic if there exists a continuous map $F:[0,1] \times[0,1] \rightarrow X$ with $F(s, 0)=\alpha(s), F(s, 1)=\beta(s)$ and $F(0, t)=F(1, t)=\gamma(t)$ for all $s$ and $t$. Unlike ordinary homotopy the curve $\gamma(t)$ is not required to be a constant curve. The relation of being freely homotopic is an equivalence relation on the set of closed curves in $X$ that are parametrized on $[0,1]$. These equivalence classes will be called free homotopy classes. A free homotopy class is nontrivial if it does not contain the constant curves. In the sequel we shall consider only nontrivial free homotopy classes. If $\alpha:[0,1] \rightarrow X$ is a continuous closed curve, then $[\alpha]$ will denote the free homotopy class to which $\alpha$ belongs.

If two closed curves $\alpha, \beta:[0,1] \rightarrow X$ have the same basepoint $p$ in $X$, then it is easy to verify that $[\alpha]=[\beta]$ if and only if the homotopy classes of $\alpha$ and $\beta$ are conjugate in $\pi_{1}(X, p)$. Hence there is a bijection between the free homotopy classes of $X$ and the conjugacy classes of $\pi_{1}(X, p)$. If $X_{1}, X_{2}$ are arc connected topological spaces and if $\theta: \pi_{1}\left(X_{1}, p_{1}\right) \rightarrow \pi_{1}\left(X_{2}, p_{2}\right)$ is an isomorphism, then $\theta$ induces a bijection between the free homotopy classes of $X_{1}$ and $X_{2}$.

The set of free homotopy classes of a space $X$ is not a group, but one can define powers and inverses of a free homotopy class; if $\alpha:[0,1] \rightarrow X$ is a closed curve with basepoint $p$, then for any integer $n \neq 0$ we may define $[\alpha]^{n}=\left[\alpha^{n}\right]$, where $\alpha \in \pi_{1}(X, p)$. This definition makes sense by the discussion of the previous paragraph.

If $X$ is expressed as a quotient space $\tilde{X} / \Gamma$, where $\tilde{X}$ is simply connected and $\Gamma$ is a freely acting, properly discontinuous group of homeomorphisms of $\tilde{X}$, then we may describe the free homotopy classes of $X$ in terms of $\Gamma$, which is isomorphic to $\pi_{1}(X)$. If $\pi: \tilde{X} \rightarrow X$ is the covering projection then for each element $\phi \neq 1$ in $\Gamma$ we define $T(\phi)=\{\pi \circ \alpha$ : $\alpha$ is a continuous curve in $\tilde{X}$ parametrized on $[0,1]$ with $\alpha(1)=\phi(\alpha(0))\}$. Note that $T(\phi)=T\left(\zeta \phi \zeta^{-1}\right)$ for any $\zeta \in \Gamma$. It is straightforward to show that $T(\phi)$ is a free homotopy class of $X$ and that all free homotopy classes of $X$ may be represented in this manner.

If $X$ is a compact $C^{2}$ Riemannian manifold, then it is well known that every nontrivial free homotopy class contains at least one smooth periodic geodesic. 
Briefly, one locates a closed curve $\gamma$ of smallest length in the free homotopy class; the existence of $\gamma$ is assured by the compactness of $M$. Then one argues that first $\gamma$ must be a geodesic and second $\gamma$ must be smoothly closed, for otherwise one could shorten $\gamma$ and remain within the free homotopy class.

Nonpositively curved manifolds. We return to our convention that $M$ denotes a compact $C^{\infty}$ manifold of nonpositive sectional curvature. For such manifolds $M$ we may sharpen the remarks above and introduce three meaningful types of free homotopy classes. Before doing so we state a fact that is well known but does not seem to be explicitly proved in the literature.

Proposition 3.1. Let $\gamma_{1}, \gamma_{2}:[0,1] \rightarrow M$ be two smooth periodic geodesics that belong to the same nontrivial free homotopy class in $M$. Then $\gamma_{1}, \gamma_{2}$ have the same length $c>0$ and there exists $a$ number $R>0$ and $a$ smooth, totally geodesic, isometric immersion $F: S^{1}(c) \times[0, R] \rightarrow M$ with $F\left(S^{1} \times\{0\}\right)=\gamma_{1}$, and $F\left(S^{1} \times\{R\}\right)=\gamma_{2}$.

\section{Proof. Appendix II.}

Here $S^{1}(c)$ denotes the circle of length $c$ parametrized on $[0,1]$. Geometrically stated, the result above asserts that there exists a flat totally geodesic cylinder in $M$ that joins $\gamma_{1}$ to $\gamma_{2}$ and whose intermediate closed curves are smooth periodic geodesics of length $c$. (It follows that if $M$ has strictly negative sectional curvature, then each free homotopy class has a unique smooth periodic geodesic representative.) For each $x \in S^{1}(c)$ the curves $t \rightarrow F(x, t)$ are unit speed geodesics of length $R$ that meet $\gamma_{1}$ and $\gamma_{2}$ orthogonally. For this reason, we refer to $R$ as the length of the flat homotopy $F$ and let $L_{F}\left(\gamma_{1}, \gamma_{2}\right)$ denote this length. The flat homotopy $F$ that joins $\gamma_{1}$ to $\gamma_{2}$ is not in general unique. However the number $c$ is the length of the periodic geodesics that represent $[\alpha]$ and is clearly independent of $F$. We refer to $c$ as the magnitude of $[\alpha]$ and denote it by $|[\alpha]|$.

A free homotopy class $[\alpha]=T(\phi)$ is primitive if there exists no free homotopy class $[\beta]$ and no integer $n \geqslant 2$ such that $[\alpha]=[\beta]^{n}$. Equivalently $[\alpha]=T(\phi)$ is primitive if $\phi$ cannot be written as $\phi=\psi^{n}$ for some $\psi \in \Gamma$ and some integer $n \geqslant 2$. Every free homotopy class $[\alpha]=[\beta]^{n}$ for some primitive free homotopy class $[\beta]$ and some integer $n \geqslant 1$; this follows since $\left|[\beta]^{n}\right|=n|[\beta]|$ and a compact Riemannian manifold does not contain periodic geodesics of arbitrarily small length.

Definition 3.2. A free homotopy class $[\alpha]$ in $M$ is Clifford if for every point $p \in M$ there exists a smooth periodic geodesic that contains $p$ and represents $[\alpha]$.

Definition 3.3. A free homotopy class $[\alpha]$ in $M$ is bounded if there exists a number $R>0$ such that $L_{F}\left(\gamma_{1}, \gamma_{2}\right) \leqslant R$ for any smooth periodic geodesics $\gamma_{1}, \gamma_{2}$ that represent $[\alpha]$ and any flat homotopy $F$ that joins $\gamma_{1}$ to $\gamma_{2}$.

Definition 3.4. A free homotopy class $[\alpha]$ in $M$ has rank 1 if there exists a smooth periodic geodesic $\gamma$ that has rank 1 and represents [ $\alpha$ ].

In view of the discussion of $\S 1$ and the proof of Proposition 3.1, it is clear that if one smooth periodic geodesic representative of $[\alpha]$ has rank 1 , then all such representatives of $[\alpha]$ have rank 1 . The next two results present equivalent formulations of the Clifford and bounded conditions for a free homotopy class in $M$. We now regard $M$ as a quotient manifold $H / \Gamma$. 
Proposition 3.5. The following are equivalent properties for a free homotopy class $[\alpha]=T(\phi)$ in $M$.

(1) $[\alpha]$ is Clifford.

(2) $\phi$ is a Clifford translation of $H$.

(3) The centralizer of $\phi$ in $\Gamma$ has finite index in $\Gamma$.

Proof. See Appendix II.

Proposition 3.6. The following are equivalent properties for a free homotopy class $[\alpha]=T(\phi)$ in $M$.

(1) $[\alpha]$ is unbounded.

(2) The centralizer of $\phi$ in $\Gamma$ is not infinite cyclic.

(3) $\phi$ is contained in a free abelian rank 2 subgroup of $\Gamma$.

(4) There exists a smooth, totally geodesic isometric imbedding $\tilde{F}: \mathbf{R}^{2} \rightarrow H$ and $a$ free abelian rank 2 subgroup $G$ of $\Gamma$ such that $\phi \in G, G$ leaves $\tilde{F}\left(\mathbf{R}^{2}\right)$ invariant, $G$ acts on $\tilde{F}\left(\mathbf{R}^{2}\right)$ by Euclidean translations and $\tilde{F}\left(\mathbf{R}^{2}\right) / G$ is a 2-dimensional torus.

(5) The class $[\alpha]$ has more than one periodic geodesic representative, and for any two distinct periodic geodesics $\gamma_{0}, \gamma_{1}$ that belong to $[\alpha]$ there are infinitely may distinct flat homotopies that join $\gamma_{0}$ to $\gamma_{1}$.

(6) There exist distinct periodic geodesics $\gamma_{0}, \gamma_{1}$ that belong to $[\alpha]$ and admit infinitely many distinct flat homotopies joining $\gamma_{0}$ to $\gamma_{1}$.

(7) The isometry $\phi$ fixes at least three distinct points in $H(\infty)$.

(8) There exists a smooth, totally geodesic, isometric immersion $F: S^{1} \times \mathbf{R} \rightarrow M$ such that $F\left(S^{1} \times\{t\}\right)$ is a smooth periodic geodesic belonging to $[\alpha]$ for every $t$.

(9) There exists a flat 2-dimensional torus $T^{2}$, not necessarily rectangular, and a smooth totally geodesic isometric immersion $F: T^{2} \rightarrow M$ such that $F$ maps one set of ruling geodesics of $T^{2}$ into smooth periodic geodesics belonging to $[\alpha]$.

Proof. Appendix II.

If $T^{2}=\mathbf{R}^{2} / G$ is the flat torus whose deckgroup $G$ is generated by translations $T_{i}: v \rightarrow v+\zeta_{i}, i=1,2$, then the projections into $T^{2}$ of the parallel pencils in $\mathbf{R}^{2}$ with directions $\zeta_{1}, \zeta_{2}$ will be called the ruling geodesics of $T^{2}$.

From conditions (5) and (6) of the preceding result and, in particular, the proof of $(6) \Rightarrow(2)$ we immediately obtain

COROllary 3.6. Let $[\alpha]=T(\phi)$ be a primitive free homotopy class. Then the following are equivalent:

(1) $[\alpha]$ is bounded.

(2) Either $[\alpha]$ has a unique periodic geodesic or for any two distinct periodic geodesics $\gamma_{0}, \gamma_{1}$ that belong to $[\alpha]$ there is a unique flat homotopy joining $\gamma_{0}$ to $\gamma_{1}$.

(3) Either $[\alpha]$ has a unique periodic geodesic or there exist two distinct periodic geodesics $\gamma_{0}, \gamma_{1}$ that belong to $[\alpha]$ and admit a unique flat homotopy joining $\gamma_{0}$ to $\gamma_{1}$.

As a corollary of the preceding results we also obtain the following 
Proposition 3.7. If $M$ admits a Clifford free homotopy class, then for every free homotopy class $[\alpha]$ there exists an integer $n \geqslant 1$ such that $[\alpha]^{n}$ is unbounded.

Proof. Appendix II. We remark that if the fundamental group of $\boldsymbol{M}$ has nontrivial center, then $M$ admits Clifford free homotopy classes by Proposition 3.5.

It is natural to compare the three types of free homotopy classes. From the results above it follows that every rank 1 class is bounded and every Clifford class is unbounded. If $[\alpha]$ is unbounded, then $[\alpha]^{n}$ is unbounded for every integer $n \neq 0$. However, the 3-manifold example of Appendix II shows that it is possible to have a bounded free homotopy class $[\alpha]$ such that $[\alpha]^{n}$ is unbounded for some integer $n \geqslant 2$. The results above also show that Clifford and bounded free homotopy classes $[\alpha]=T(\phi)$ have a simple description in terms of algebraic properties of the conjugacy class of $\phi$ in $\pi_{1}(M)$. As the discussion later in this section shows there does not seem to be any such simple algebraic criterion for a free homotopy class to have rank 1. Nevertheless the main theorem of the next section shows that there is a close relationship between the rank 1 condition for a free homotopy class and algebraic properties of the fundamental group of $M$.

As we shall see below a rank 1 free homotopy class is basically different from its closest relative, a bounded free homotopy class, in all dimensions $>4$. Nevertheless, it is illuminating to express this difference in the following way suggested by R. Bishop. Let $M=H / \Gamma$ be compact and let $[\alpha]=T(\phi)$ be a given free homotopy class. Let $\gamma$ be a geodesic of $H$ that is translated by $\phi$ and let $p=\gamma\left(t_{0}\right)$ be a point on $\gamma$. Let $A_{p} \subseteq T_{p} H$ be the set of vectors $w$ orthogonal to $\gamma^{\prime}\left(t_{0}\right)$ such that $\gamma_{w}[0, \infty)$ is contained in a flat half-plane bounded by $\gamma$. Let $S: T_{p} H \rightarrow T_{p} H$ be the linear isometry given by $S(w)=\left(P \circ \phi_{*}\right)(w)$ where $P$ is parallel translation along $\gamma$ from $\gamma(c)=(\phi \circ \gamma)\left(t_{0}\right)$ to $\gamma\left(t_{0}\right)=p$. It is easy to see that $S$ leaves $A_{p}$ invariant. One can now show without difficulty that $[\alpha]=T(\phi)$ has rank 1 if and only if $A_{p}$ is empty and $[\alpha]$ is bounded if and only if $S$.has no fixed point in $A_{p}$.

The structure of the sets $A_{p}$ is unclear. In general, $A_{p}$ is not a vector space and may not even be invariant under the map $w \rightarrow-w, w \in T_{p} H$. Although the set $A_{p}$ depends on the geodesic $\gamma$ and the point $p$ of $\gamma$ one can show that $B_{\phi}=\left\{\gamma_{w}(\infty)\right.$ : $\left.w \in A_{p}\right\}$ is a subset of $H(\infty)$ that depends neither on $\gamma$ nor on $p$, only on $\phi$.

Before making further comparisons, we consider the rank 1 property alone. If $M$ is a Visibility manifold, then every free homotopy class [ $\alpha]$ of $M$ is bounded by Theorem 4.1 of [7]. The truth of the converse assertion is unknown except in dimension 2. If a 2-dimensional Hadamard manifold $H$ admits a flat half-plane and also a compact quotient $H / \Gamma$, then it is easy to see that $H$ is flat. Hence for a compact 2-manifold $M$ the properties of admitting a rank 1 geodesic, satisfying the Visibility axiom and having all nontrivial free homotopy classes bounded are equivalent.

For compact manifolds $M$ of dimension $\geqslant 3$ the rank 1 property may hold when the Visibility axiom fails as the examples in [1, pp. 52-53] and [15, pp. 228-229] show. The next result shows that in dimension 3 there is still a close relationship between rank 1 and bounded free homotopy classes. The 3-manifold example in Appendix II is also an illustration of case (3). 
Proposition 3.8. Let $M$ be a compact 3-manifold. Then exactly one of the following cases occurs:

(1) $M$ is flat. There exists an integer $n \geqslant 1$ such that $[\alpha]^{n}$ is Clifford for every free homotopy class $[\alpha]$.

(2) $M$ has rank 1. A free homotopy class $[\alpha]$ has rank 1 if and only if $[\alpha]^{n}$ is bounded for every integer $n \geqslant 1$.

(3) $M$ is not flat and no free homotopy class has rank 1 . Then

(a) for each class $[\alpha]$ there exists an integer $n \geqslant 1$ such that $[\alpha]^{n}$ is unbounded.

(b) There exists a unique (up to inverse) free homotopy class [ $\beta$ ] such that the Clifford classes of $M$ are precisely the powers of $[\beta]$. There exist nontrivial free homotopy classes $[\alpha]$ in $M$ such that $[\alpha]^{n}$ is not Clifford for any integer $n>1$.

(c) $M$ admits a covering $M^{*}$ of multiplicity $<2$ such that $\pi_{1}\left(M^{*}\right)$ has nontrivial center. Moreover $M^{*}$ admits a finite covering $M^{* *}$ such that any finite covering $\tilde{M}$ of $M^{* *}$ is diffeomorphic to a product $S^{1} \times M_{g}$ where $M_{g}$ is a compact orientable surface of genus $g \geqslant 2 . \tilde{M}$ is not necessarily isometric to the Riemannian product of $S^{1}$ and $M_{g}$ relative to any metric of nonpositive curvature on $M_{g}$.

\section{Proof. Appendix II.}

An immediate consequence of this result is

COROLlaRY 3.8. Let $M$ be a compact 3-manifold. Then a free homotopy class $[\alpha]$ has rank 1 if and only if $[\alpha]^{n}$ is bounded for every integer $n>1$.

This characterization of rank 1 classes fails in higher dimensions as the final result of this section shows.

Proposition 3.9. Let $H$ denote the Riemannian product of two copies of the hyperbolic plane, each equipped with the usual Poincare metric of $K \equiv-1$. Let $\Gamma$ be an irreducible uniform lattice in $H$, and let $M=H / \Gamma$. Then

(1) every free homotopy class $[\alpha]$ fails to have rank 1.

(2) There exist free homotopy classes $[\alpha]$ such that $[\alpha]^{n}$ is bounded for every integer $n \geqslant 1$.

(3) There exists a covering $M^{*}$ of multiplicity $\leqslant 2$ such that for any free homotopy class $[\alpha]$ in $M^{*}$ either $[\alpha]$ is unbounded or $[\alpha]^{n}$ is bounded for all $n>1$. Classes of both types exist.

\section{Proof. Appendix II.}

The existence of uniform irreducible lattices in the space $H$ above is classically known. See for example [24, p. 64]. In general if $n \geqslant 4$ is any integer let $H$ be the Riemannian product of a hyperbolic plane with $K \equiv-1$ and a hyperbolic space with $K \equiv-1$ and dimension $n-2$. The paper [3] demonstrates the existence of uniform irreducible lattices $\Gamma$ in $H$, and the proof of the result just stated carries over to show that properties (1) and (2) are still valid for $M=H / \Gamma$ in the general situation. 
4. Rigidity theorems. Our main result of this section and of the paper is

THEOREM A. Let $M, M^{*}$ denote compact $C^{\infty}$ manifolds of nonpositive sectional curvature. Let $\theta: \pi_{1}(M, p) \rightarrow \pi_{1}\left(M^{*}, p^{*}\right)$ be an isomorphism, and let $\theta$ also denote the induced bijection of free homotopy classes. If $[\alpha]$ denotes an arbitrary free homotopy class in $M$, then

(1) $[\alpha]$ is Clifford if and only if $\theta[\alpha]$ is Clifford.

(2) $[\alpha]$ is bounded if and only if $\theta[\alpha]$ is bounded.

(3) $[\alpha]$ has rank 1 if and only if $\theta[\alpha]$ has rank 1 .

It follows from Satz 2.7 of [1] that $M$ has rank 1 if and only if $M$ admits a free homotopy class $[\alpha]$ of rank 1 . From this fact and the theorem we immediately obtain the next two results.

Corollary 1. Let $M, M^{*}$ be compact $C^{\infty}$ manifolds of nonpositive sectional curvature with isomorphic fundamental groups. Then $M$ has rank 1 if and only if $M^{*}$ has rank 1.

COROllary 2. Let $M$ be a compact $C^{\infty}$ manifold, and let $g_{1}, g_{2}$ be any two $C^{\infty}$ metrics in $M$ of nonpositive sectional curvature. Let $[\alpha]$ be any free homotopy class in $M$. Then

(1) $[\alpha]$ has rank 1 relative to $g_{1}$ if and only if $[\alpha]$ has rank 1 relative to $g_{2}$.

(2) $[\alpha]$ is bounded (Clifford) relative to $g_{1}$ if and only if $[\alpha]$ is bounded (Clifford) relative to $g_{2}$.

(3) $\left(M, g_{1}\right)$ has rank 1 if and only if $\left(M, g_{2}\right)$ has rank 1 .

REMARK. Theorem A and its corollaries are useful only when there is enough zero sectional curvature to admit unbounded or Clifford free homotopy classes. If $M$ has strictly negative sectional curvature, for example, then every free homotopy class in $M$ is bounded and Theorem A asserts only that every free homotopy class in $M^{*}$ is bounded. In this case, however, $M$ is a Visibility manifold and Theorem $\mathrm{C}$ of the next section proves with less work the stronger result that $M^{*}$ also is a Visibility manifold. If unbounded or Clifford free homotopy classes do exist in $M$, then Theorem A has the extra effect of placing restrictions on the automorphism group of $\pi_{1}(M)$.

For 3-manifolds an isomorphism of fundamental groups is in some cases almost induced by a diffeomorphism. We do not know if the next result is valid in the rank 1 case.

THEOREM B. Let $M_{1}, M_{2}$ be compact $C^{\infty}$ 3-manifolds of nompositive sectional curvature and isomorphic fundamental groups. If one (hence both) of the manifolds does not have rank 1 , then there exist an integer $n \geqslant 1$ and coverings $M_{1}^{*}, M_{2}^{*}$ of $M_{1}, M_{2}$ of multiplicity $n$ such that $M_{1}^{*}$ is diffeomorphic to $M_{2}^{*}$.

We now prove Theorem A. Parts (1) and (2) are immediate consequences of Propositions 3.5 and 3.6, which characterize Clifford and bounded free homotopy 
classes in terms of algebraic conditions on the fundamental group that are clearly preserved by isomorphisms. The proof of part (3) appears to have no such easy solution in view of the discussion of the previous section. The proof of part (3) consists of a series of rather technical lemmas followed by a short argument. To clarify the exposition we present the final argument here, but merely state the lemmas. Proofs of the lemmas are found in Appendix III.

Before stating the lemmas to Theorem $\mathrm{A}$ we need to define Hausdorff distance. If $B$ is a set in a metric space $X$ and $r>0$ is any number, we define $T_{r}(B)=\{p \in$ $X: d(p, B) \leqslant r\}$, where $d(p, B)=\inf \{d(p, q): q \in B\}$. If $A, B$ are any two subsets of $X$ then their Hausdorff distance $\operatorname{Hd}(A, B)$ is defined to be $\inf \left\{r>0: B \subseteq T_{r}(A)\right.$ and $\left.A \subseteq T_{r}(B)\right\}$. It is clear that $\operatorname{Hd}(A, B)=\operatorname{Hd}(B, A)$ and $\operatorname{Hd}(A, C)<\operatorname{Hd}(A, B)$ $+\operatorname{Hd}(B, C)$ for any subsets $A, B, C$ of $X$.

Next we establish some notation common to all of the lemmas. Express $M, M^{*}$ as quotient manifolds $H / \Gamma, H^{*} / \Gamma^{*}$, respectively, and let $\theta: \Gamma \rightarrow \Gamma^{*}$ be an isomorphism obtained in the manner of $\$ 2$ from the given isomorphism $\theta: \pi_{1}(M, p) \rightarrow$ $\pi_{1}\left(M^{*}, p^{*}\right)$. If $\phi \in \Gamma$ is given let $\phi^{*}$ denote $\theta(\phi) \in \Gamma^{*}$. By Propositions 2.1 and 2.2 we can construct a $\theta$-equivariant $(\zeta, r)$ pseudoisometry $F: H \rightarrow H^{*}$ for some positive constants $\zeta, r$. Recall that $T\left(\phi^{*}\right)=\theta[T(\phi)]$ for every $\phi \in \Gamma$ in the notation of $\S 3$. Here $\theta$ denotes the induced bijection on free homotopy classes.

Every element of $\Gamma$ or $\Gamma^{*}$ is hyperbolic since the quotient manifolds $H / \Gamma$ and $H^{*} / \Gamma^{*}$ are compact. Given an element $\phi \neq 1$ in $\Gamma$ let $\gamma$ denote a fixed geodesic translated by $\phi$ with periodic $\omega=\inf d_{\phi}$. Let $\gamma^{*}$ denote a fixed geodesic translated by $\phi^{*}$ with period $\omega^{*}=\inf d_{\phi^{*}}$.

LEMMA A1. $\operatorname{Hd}\left(F(\gamma \mathbf{R}), \gamma^{*} \mathbf{R}\right) \leqslant c$ for some $c>0$.

LEMMA A2. Let $\sigma$ be a maximal geodesic in $H$ equivalent to $\gamma$, and let $c>0$ be the constant of Lemma A1. Then for every $t \in \mathbf{R}$ we have $d\left(F(\sigma t), \gamma^{*}\right)>$ $\zeta \operatorname{Hd}(\sigma(\mathbf{R}), \gamma(\mathbf{R}))-c$, provided that $\operatorname{Hd}(\sigma(\mathbf{R}), \gamma(\mathbf{R}))>r$, where $\zeta, r$ are the constants appearing in the definition of $F$.

LemMA A3. Let $\sigma$ be a maximal geodesic in $H$ equivalent to $\gamma$. Then:

(1) There exists a sequence $\left\{n_{k}\right\}$ of integers that diverges to $+\infty$ such that $\operatorname{Hd}\left(\sigma(\mathbf{R}), \phi^{n_{k}} \circ \sigma(\mathbf{R})\right) \rightarrow 0$ as $k \rightarrow+\infty$.

(2) Let $\gamma^{*}$ be parametrized so that $\gamma^{*}(0)$ is the foot (= unique closest point) on $\gamma^{*}$ of $(F \circ \sigma)(0)$. If $s_{k}$ is the number such that $\gamma^{*}\left(s_{k}\right)$ is the foot on $\gamma^{*}$ of $(F \circ \sigma)\left(n_{k} \omega\right)$, then $\left|s_{k}-n_{k} \omega^{*}\right| \rightarrow 0$ as $k \rightarrow+\infty$.

LEMMA A4. Let $\beta$ be a maximal geodesic in an arbitrary Hadamard manifold $H$, and let $\beta$ be translated by some element $\phi \neq 1$ in $I(H)$. Suppose that $\beta$ bounds no flat half-plane in $H$. Then given any number $K>1$ we can find positive numbers $R^{*}, A^{*}$ (depending on $K, \phi, \gamma$ ) with the following property:

Let $\sigma:[a, b] \rightarrow H$ be a continuous, piecewise $C^{\infty}$ curve such that $d(\sigma, \beta)>R^{*}$ and $d(P \sigma a, P \sigma b) \geqslant A^{*}$, where $P: H \rightarrow \beta$ is the orthogonal projection onto $\beta$. Then the length of $\sigma=L(\sigma) \geqslant K d(P \sigma a, P \sigma b)$. 
We refer to Appendix III for the proofs of the lemmas. The projection map $P$ : $H \rightarrow \beta$ sends a point $p \in H$ to its foot or unique closest point on $\beta$. It has the property that $d(P p, P q) \leqslant d(p, q)$ for all points $p, q$ in $H$ and all geodesics $\beta$. For further details see Lemma 3.2 of [2].

We are now ready to prove part (3) of Theorem A. It clearly suffices to show that if $\theta[\alpha]$ has rank 1 , then $[\alpha]$ has rank 1 . Let $[\alpha]$ be a free homotopy class such that $\theta[\alpha]$ has rank 1 and choose an element $\phi \in \Gamma$ so that $T(\phi)=[\alpha]$. Then $T\left(\phi^{*}\right)=\theta[\alpha]$ where $\phi^{*}=\theta(\phi)$. Let $\gamma, \gamma^{*}$ be geodesics translated by $\phi, \phi^{*}$ with periods $\omega, \omega^{*}$. Let $F: H \rightarrow H^{*}$ be a $(\zeta, r)$ pseudoisometry for some positive constants $\zeta, r$ such that $F(\phi p)=\phi^{*} F(p)$ for all $p \in H$ and all $\phi \in \Gamma$. Let $c>0$ be the constant given by Lemma A1.

Since $F$ is uniformly continuous there exists a number $B>0$ such that $d(F p, F q)$ $\langle B d(p, q)$ whenever $d(p, q) \geqslant \omega$ for any points $p, q$ in $H$. Now fix a number $K>\max \left\{1,\left(B \omega / \omega^{*}\right)\right\}$. Since $\theta[\alpha]$ has rank 1 by hypothesis there is no flat half-plane in $H^{*}$ with boundary $\gamma^{*}$. Hence we may choose positive numbers $R^{*}, A^{*}$ as in Lemma A4 to correspond to $\phi^{*}, \gamma^{*}$ and $K$. We assert that if $\sigma$ is any geodesic in $H$ equivalent to $\gamma$, then $\operatorname{Hd}(\sigma, \gamma) \leqslant r+(1 / \zeta)\left(R^{*}+c+B \omega\right)$. From this it will follow that $\gamma$ bounds no flat half-plane in $H$ and, hence, that $[\alpha]$ is a free homotopy class of rank 1.

Let $\sigma$ be a maximal geodesic of $H$ that is equivalent to $\gamma$, and let $D$ denote $\operatorname{Hd}(\sigma, \gamma)$. We may assume that $D \geqslant r$ for otherwise we are already done. Parametrize $\gamma^{*}$ so that $P(F \sigma 0)=\gamma^{*}(0)$, where $P: H^{*} \rightarrow \gamma^{*}$ is orthogonal projection. For each integer $i \geqslant 0$ let $x_{i}=\sigma(i \omega)$ and let $\sigma_{i}^{*}$ denote the geodesic segment in $H^{*}$ from $F\left(x_{i}\right)$ to $F\left(x_{i+1}\right)$. For each integer $k \geqslant 1$ let $\delta_{k}$ be the geodesic polygonal arc consisting of the union of the geodesic segments $\sigma_{i}^{*}, 0<i<-1+n_{k}$, where $\left\{n_{k}\right\}$ is the divergent sequence of integers whose existence is given by Lemma A3. Now $L\left(\sigma_{i}^{*}\right)=d\left(F x_{i}, F x_{i+1}\right) \leqslant B d\left(x_{i}, x_{i+1}\right)=B \omega$ by the way in which $B$ was chosen. Moreover $d\left(F x_{i}, \gamma^{*}\right) \geqslant \zeta D-c$ for every $i$ by Lemma A2, and hence it follows that $d\left(\delta_{k}, \gamma^{*}\right) \geqslant \zeta D-c-B \omega$ for every integer $k \geqslant 1$.

Suppose now that $D>r+(1 / \zeta)\left(R^{*}+c+B \omega\right)$. Then $d\left(\delta_{k}, \gamma^{*}\right)>R^{*}$ and $d\left(P F \sigma 0\right.$, PFon $\left._{k} \omega\right)=d\left(\gamma^{*} 0, \gamma^{*} s_{k}\right)=s_{k} \rightarrow+\infty$ as $k \rightarrow+\infty$ by Lemma A3. We may now apply Lemma A4 to conclude that $L\left(\delta_{k}\right)>K d\left(P F \sigma 0, P F \sigma n_{k} \omega\right)=K s_{k}$. On the other hand we have

$$
L\left(\delta_{k}\right)=\sum_{i=0}^{-1+n_{k}} L\left(\sigma_{i}^{*}\right) \leqslant B \omega n_{k}
$$

since $L\left(\sigma_{i}^{*}\right)<B \omega$ for every $i$. We obtain the inequalities $K s_{k}<L\left(\delta_{k}\right)<B \omega n_{k}$. Since $\left|s_{k}-n_{k} \omega^{*}\right| \rightarrow 0$ as $k \rightarrow+\infty$ by Lemma A3, it follows that $K<$ $\lim _{k \rightarrow \infty}\left(B \omega n_{k} / s_{k}\right)=B \omega / \omega^{*}$, contradicting the definition of $K$. Therefore $\operatorname{Hd}(\sigma, \gamma)$ $=D<r+(1 / \zeta)\left(R^{*}+c+B \omega\right)$, which completes the proof of part (3) of Theorem A.

We now prove Theorem B. Let $M_{1}, M_{2}$ be as in the statement of Theorem B. If $M_{1}$ is flat then the Bieberbach theorems state that $\pi_{1}\left(M_{1}\right)$ contains a normal abelian subgroup $T_{1}$ of finite index $n$, and that the covering $M_{1}^{*}$ of $M_{1}$ with 
multiplicity $n$ whose fundamental group is $T_{1}$ is a flat torus [28, p. 100]. Since $\pi_{1}\left(M_{2}\right)$ is isomorphic to $\pi_{1}\left(M_{1}\right)$ there exists a normal abelian subgroup $T_{2} \subseteq$ $\pi_{1}\left(M_{2}\right)$ of index $n$. Expressing $M_{2}$ as a quotient $H_{2} / \Gamma_{2}$ and considering $T_{2}$ as a normal abelian subgroup of $\Gamma_{2}$ of index $n$, it follows from Proposition 2.3 of [5] that $T_{2}$ consists of Clifford translations of $H_{2}$. Let $M_{2}^{*}$ be the covering of $M_{2}$ of multiplicity $n$ whose fundamental group is $T_{2}$. Then $M_{2}^{*}$ is a flat torus by Theorem 2 of [27], and hence $M_{1}^{*}$ is diffeomorphic to $M_{2}^{*}$ since they are tori of the same dimension.

Next suppose that $M_{1}$ is not flat and does not have rank 1. By part (c) of Proposition 3.8 we can find for $i=1,2$ finite coverings $M_{i}^{* *}$ of $M_{i}$ with the property that any finite covering $\tilde{M}_{i}$ of $M_{i}^{* *}$ is diffeomorphic to a product $S^{1} \times M_{g_{i}}$, where $M_{g_{i}}$ is a compact orientable surface of genus $g_{i} \geqslant 2$. If $M_{i}=$ $H_{i} / \Gamma_{i}$ for $i=1,2$ let $G_{i} \subseteq \Gamma_{i}$ be the subgroup of finite index such that $M_{i}^{* *}=$ $H_{i} / G_{i}$.

By hypothesis there exists an isomorphism $\theta: \Gamma_{1} \rightarrow \Gamma_{2}$. Let $\tilde{G}_{1}=G_{1} \cap \theta^{-1}\left(G_{2}\right)$ and let $\tilde{G}_{2}=\theta\left(\tilde{G}_{1}\right)$. Then $\tilde{G}_{i}$ is a subgroup of finite index in $G_{i}$, and hence if $\tilde{M}_{i}=H_{i} / \tilde{G}_{i}$, then $\tilde{M}_{i}$ is a finite cover of $M_{i}^{* *}$. By the choice of $M_{i}^{* *}$ we see that $\tilde{M}_{i}$ is diffeomorphic to $S^{1} \times M_{g_{i}}$ for $i=1,2$, where $M_{g}$ is a compact orientable surface of genus $g_{i} \geqslant 2$. Since the fundamental groups $\tilde{G}_{i}$ of $\tilde{M}_{i}$ are isomorphic for $i=1,2$ it follows that $g_{1}=g_{2}$ and hence $\tilde{M}_{1}$ is diffeomorphic to $\tilde{M}_{2}$. This completes the proof since $\tilde{M}_{i}$ is a finite covering of $M_{i}$ for $i=1,2$.

5. Visibility manifolds. We observed earlier that any compact Visibility manifold has rank 1. If $M, M^{*}$ are compact manifolds of nonpositive sectional curvature with isomorphic fundamental groups and if $M$ is a Visibility manifold, then the result of the previous section says that $M^{*}$ has rank 1 . However, we can say more.

TheOREM C. Let $M, M^{*}$ be compact $C^{\infty}$ manifolds of nonpositive sectional curvature with isomorphic fundamental groups. If $M$ is a Visibility manifold, then $M^{*}$ is also a Visibility manifold.

Corollary 3. Let $M$ be a compact $C^{\infty}$ manifold, and let $g_{1}, g_{2}$ be two metrics of nonpositive sectional curvature on $M$. If $\left(M, g_{1}\right)$ is a Visibility manifold, then $\left(M, g_{2}\right)$ is a Visibility manifold.

The corollary is contained in Theorem 5.1 of [7]. The proof of that result carries over to give a proof of Theorem $\mathrm{C}$ once we have established the following geodesic comparison result, which is an extension of Proposition 5.5 of [7]. The idea is originally due to Morse [21] and has also been used in [7], [14], [17], [22]. We remark that by the discussion in [7, p. 153] the Visibility and uniform Visibility axioms are equivalent if $H$ admits a compact quotient manifold.

Proposition. Let $H^{*}$ be a uniform Visibility manifold, and let $F: H \rightarrow H^{*}$ be a $(\zeta, r)$ pseudoisometry for some positive constants $\zeta, r$. Then there exists a constant $R>0$ such that if $\sigma:[a, b] \rightarrow H$ is a geodesic segment of length $>r$ and if $\sigma^{*}$ is the geodesic segment in $H^{*}$ joining the endpoints of $F \circ \sigma$, then $\operatorname{Hd}\left(F \circ \sigma[a, b], \sigma^{*}\right)<R$. 
Proof. Let $F, H, H^{*}$ be as above. We show first that there exists a constant $R^{*}>0$ such that if $\sigma:[a, b] \rightarrow H$ is a geodesic segment of length $>r$ and if $\sigma^{*}$ is the geodesic segment in $H^{*}$ joining the endpoints of $F \circ \sigma$, then $d\left(F(\sigma t), \sigma^{*}\right)<R^{*}$ for every $t \in[a, b]$.

Assuming for the moment that such a constant $R^{*}$ has been found we prove the proposition. By the uniform continuity of $F$ we may choose a constant $R_{0}>0$ such that $d(F p, F q) \leqslant R_{0}$ if $d(p, q) \leqslant r$. We may also choose $k^{*}>0$ so that $d(F p, F q)$ $\leqslant k^{*} d(p, q)$ whenever $d(p, q) \geqslant r$. Let $\alpha=\max \left\{k^{*} r, R_{0}\right\}$ and let $R=3 R^{*}+\alpha$. We claim that $R$ satisfies the assertion of the proposition.

Let $\sigma:[a, b] \rightarrow H$ have length $\geqslant r$ and let $N \geqslant 1$ be the integer such that $N r \leqslant L(\sigma)<(N+1) R$. Let $q_{0}=\sigma(a), q_{N+1}=\sigma(b)$ and $q_{k}=\sigma(a+k r), 0<k<$ $N$. By the assertion of the first paragraph of the proof we can choose $p_{k} \in \sigma^{*}$ so that $p_{0}=F(\sigma a), p_{N+1}=F(\sigma b)$ and $d\left(p_{k}, F q_{k}\right) \leqslant R^{*}$ for every $0<k<N+1$. By the definition of $\alpha$ we see that $d\left(F q_{k}, F q_{k+1}\right)<\alpha$ for $0<k<N$. By the triangle inequality it follows that $d\left(p_{k}, p_{k+1}\right) \leqslant 2 R^{*}+\alpha$ for $0 \leqslant k \leqslant N$. It is easy to see that every point $p$ of $\sigma^{*}$ must lie between $p_{k}$ and $p_{k+1}$ for some $k$. Hence

$$
\begin{aligned}
d(p, F \circ \sigma[a, b]) & \leqslant d\left(p, F q_{k}\right) \leqslant d\left(p, p_{k}\right)+d\left(p_{k}, F q_{k}\right) \\
& \leqslant d\left(p_{k}, p_{k+1}\right)+d\left(p_{k}, F q_{k}\right) \leqslant 3 R^{*}+\alpha=R .
\end{aligned}
$$

Since $p$ was arbitrary we have $\operatorname{Hd}\left(\sigma^{*}, F \circ \sigma[a, b]\right) \leqslant R$.

We now construct the constant $R^{*}>0$ with the properties asserted in the first paragraph of the proof. The construction will consist of a series of lemmas. First we establish some notation. Let $A>0$ be the constant of Lemma 5.6 of [7]. Since $F$ is uniformly continuous we may choose a constant $k^{*}>0$ such that if $d(p, q) \geqslant r$ then $d(F p, F q) \leqslant k^{*} d(p, q)$. We may also choose $R_{0}$ such that if $d(p, q)<r$, then $d(F p, F q) \leqslant R_{0}$. Let $\alpha=\max \left\{R_{0}, k^{*} r\right\}$.

LEMMA 1. Let $A>0$ be as above and let $\gamma^{*}$ be a maximal geodesic of $H^{*}$. For any constant $B>0$ there exists a constant $R=R(B)>0$ such that if $\sigma:[a, b] \rightarrow H^{*}$ is any continuous, piecewise $C^{\infty}$ curve satisfying

(1) $d\left(\sigma, \gamma^{*}\right) \geqslant R$,

(2) $d\left(\sigma a, \gamma^{*}\right) \leqslant R+\alpha, d\left(\sigma b, \gamma^{*}\right) \leqslant R+\alpha$,

(3) $d(P \sigma a, P \sigma b) \geqslant 2 A$,

then $L(\sigma) \geqslant B d(\sigma a, \sigma b)$.

Proof. This is a slight modification of Lemma 5.9 of [7] and is proved in the same way.

LEMMA 2. Let $B>\left(k^{*} / \zeta\right)+\left(R_{0} / \zeta r\right)$ be fixed, and let $R=R(B)>0$ be the constant of the previous lemma. Let $\sigma:[a, b] \rightarrow H$ be a geodesic segment of length $\geqslant r$, and let $\sigma^{*}$ be a maximal geodesic in $H^{*}$ such that

(1) $d\left(F \circ \sigma, \sigma^{*}\right) \geqslant R+\alpha$ and

(2) $d\left(F(\sigma a), \sigma^{*}\right)=d\left(F(\sigma b), \sigma^{*}\right)=R+\alpha$.

Then $d(P(F \sigma a), P(F \sigma b))<2 A$. 
Proof. Let $\sigma:[a, b] \rightarrow H$ be a geodesic segment of length $\geqslant r$, and let $n>1$ be the integer such that $n r \leqslant d(\sigma a, \sigma b)<(n+1) r$. Partition $\sigma$ into points $x_{0}=\sigma(a)$, $x_{1}, \ldots, x_{n+1}=\sigma(b)$ with

(1) $d(\sigma a, \sigma b)=\sum_{i=0}^{n} d\left(x_{i}, x_{i+1}\right)$ and

(2) $d\left(x_{i}, x_{i+1}\right)=r$ for $0 \leqslant i \leqslant n-1, d\left(x_{n}, x_{n+1}\right)<r$. Let $\sigma_{i}^{*}$ be the geodesic segment from $F\left(x_{i}\right)$ to $F\left(x_{i+1}\right)$ for $0 \leqslant i \leqslant n$, and let $\beta$ be the union of these segments, a geodesic polygonal arc from $F(\sigma a)$ to $F(\sigma b)$.

We assert that $L(\beta) \leqslant(n+1) \alpha$ and $d\left(\beta, \sigma^{*}\right) \geqslant R$. We show first that $L\left(\sigma_{i}^{*}\right) \leqslant$ $\alpha$ for $0 \leqslant i \leqslant n$, which will also show that $L(\beta) \leqslant(n+1) \alpha$. Now $L\left(\sigma_{i}^{*}\right)=$ $d\left(F x_{i}, F x_{i+1}\right) \leqslant k^{*} d\left(x_{i}, x_{i+1}\right)=k^{*} r$ if $0 \leqslant i \leqslant n-1$. Also $L\left(\sigma_{n}^{*}\right)=d\left(F x_{n}, F x_{n+1}\right)$ $\leqslant R_{0}$ since $d\left(x_{n}, x_{n+1}\right)<r$. Hence $L\left(\sigma_{i}^{*}\right) \leqslant \max \left\{k^{*} r, R_{0}\right\}=\alpha$ for every $i$. To show that $d\left(\beta, \sigma^{*}\right) \geqslant R$ we begin with the fact that $d\left(F x_{i}, \sigma^{*}\right)>R+\alpha$ for every $i$ by hypothesis. Since the endpoints of $\sigma_{i}^{*}$ lie at a distance $>R+\alpha$ from $\sigma^{*}$ and $L\left(\sigma_{i}^{*}\right) \leqslant \alpha$, it follows that every point of $\sigma_{i}^{*}$ lies at a distance $\geqslant R$ from $\sigma^{*}$. The same is therefore true for $\beta$ since $\beta$ is the union of the segments $\sigma_{i}^{*}$.

We summarize. The endpoints of $\beta$ are $F(\sigma a)$ and $F(\sigma b)$. The curve $\beta$ is continuous and piecewise $C^{\infty}, d\left(\beta, \sigma^{*}\right)>R$ and $d\left(F(\sigma a), \sigma^{*}\right)=R+\alpha=$ $d\left(F(\sigma b), \sigma^{*}\right)$. Suppose now that $d(P(F \sigma a), P(F \sigma b)) \geqslant 2 A$. Then by applying Lemma 1 to $\beta$ we obtain

$$
\sum_{i=0}^{n} d\left(F x_{i}, F x_{i+1}\right)=L(\beta) \geqslant B d(F \sigma a, F \sigma b) .
$$

Next observe that

$$
\begin{aligned}
d(\sigma a, \sigma b) & =\sum_{i=0}^{n} d\left(x_{i}, x_{i+1}\right) \geqslant \sum_{i=0}^{n-1} d\left(x_{i}, x_{i+1}\right) \\
& \geqslant \frac{1}{k^{*}} \sum_{i=0}^{n-1} d\left(F x_{i}, F x_{i+1}\right) \quad\left(\text { since } d\left(x_{i}, x_{i+1}\right)=r\right) \\
& =\left(1 / k^{*}\right) L(\beta)-\left(1 / k^{*}\right) d\left(F x_{n}, F x_{n+1}\right) \\
& \geqslant\left(B / k^{*}\right) d(F \sigma a, F \sigma b)-\left(R_{0} / k^{*}\right) \quad\left(\text { since } d\left(x_{n}, x_{n+1}\right)<r\right) \\
& \geqslant\left(B \zeta / k^{*}\right) d(\sigma a, \sigma b)-\left(R_{0} / k^{*}\right) \quad(\text { since } d(\sigma a, \sigma b) \geqslant r) .
\end{aligned}
$$

Hence we obtain

$$
\left(R_{0} / k^{*}\right) \geqslant\left[\left(B \zeta / k^{*}\right)-1\right] d(\sigma a, \sigma b) \geqslant\left[\left(B \zeta / k^{*}\right)-1\right] r,
$$

which contradicts the fact that $B>\left(k^{*} / \zeta\right)+\left(R_{0} / \zeta r\right)$ by hypothesis. Therefore, $d(P(F \sigma a), P(F \sigma b))<2 A$, which completes the proof of Lemma 2.

LEMMA 3. Let $B$ and $R=R(B)$ be as in Lemma 2. Let $R^{*}$ be the maximum of the two numbers $R+\alpha+R_{0}$ and $R+\alpha+(2 \alpha / r \xi)(2 R+2 \alpha+2 A)$. Let $\sigma:[a, b] \rightarrow H$ be any geodesic segment of length $>r$, and let $\sigma^{*}$ be the geodesic segment in $H^{*}$ from $F(\sigma a)$ to $F(\sigma b)$. Then for any $a \leqslant t \leqslant b$ we have $d\left(F(\sigma t), \sigma^{*}\right) \leqslant R^{*}$.

Proof. Let $\sigma:[a, b] \rightarrow H$ and $\sigma^{*}$ be as above. It suffices to show that if $d\left(F \sigma t, \sigma^{*}\right)>R+\alpha+R_{0}$ for some $t \in(a, b)$, then $d\left(F \sigma t, \sigma^{*}\right)<R+\alpha+$ $(2 \alpha / r \zeta)(2 R+2 \alpha+2 A)$. Suppose now that there exists $t \in(a, b)$ such that if 
$x=\sigma t$ then $d\left(F x, \sigma^{*}\right)>R+\alpha+R_{0}$. Let $y=\sigma\left(t_{0}\right)$ and $z=\sigma\left(u_{0}\right), t_{0}<t<u_{0}$, be the first points of $\sigma$ on opposite sides of $x$ such that $d\left(F y, \sigma^{*}\right)=d\left(F z, \sigma^{*}\right)=R+$ $\alpha$. We now proceed in several steps.

Step 1. $d(F y, F z) \geqslant \zeta d(y, z)$.

Proof. Observe that $d(F x, F y)>R_{0}$ since

$$
R+\alpha+R_{0}<d\left(F x, \sigma^{*}\right) \leqslant d(F x, F y)+d\left(F y, \sigma^{*}\right)=d(F x, F y)+R+\alpha .
$$

Hence $d(x, y) \geqslant r$ by the choice of $R_{0}$, and it follows that $d(y, z)=d(y, x)+$ $d(x, z) \geqslant d(y, x) \geqslant r$. Therefore $d(F y, F z) \geqslant \zeta d(y, z)$ since $F$ is a $(\zeta, r)$ pseudoisometry.

Step 2. $d(F x, F y) \leqslant(2 \alpha / r \zeta) d(F y, F z)$.

Proof. Partition $\sigma \mid\left[t_{0}, t\right]$ into points $x_{0}=\sigma\left(t_{0}\right), x_{1}, \ldots, x_{n+1}=\sigma(t)$, where $n r<$ $d(x, y)=d\left(\sigma t_{0}, \sigma t\right)<(n+1) r, d(x, y)=\sum_{i=0}^{n} d\left(x_{i}, x_{i+1}\right), d\left(x_{i}, x_{i+1}\right)=r$ for $0<i$ $\leqslant n-1$ and $d\left(x_{n}, x_{n+1}\right)<r$. Let $\beta$ be the geodesic polygonal curve that is the union of those segments $\sigma_{i}^{*}$ joining $F\left(x_{i}\right)$ to $F\left(x_{i+1}\right), 0 \leqslant i<n$. By the argument of Lemma 2 we have $L(\beta) \leqslant(n+1) \alpha$. Since $\beta$ joins $F x$ to $F y$ we have

$$
\begin{aligned}
d(F x, F y) & \leqslant L(\beta) \leqslant(n+1) \alpha=(n r)[(n+1) \alpha / n r] \leqslant(2 \alpha / r)(n r) \\
& \leqslant(2 \alpha / r) d(x, y) \leqslant(2 \alpha / r) d(y, z) \leqslant(2 \alpha / r \zeta) d(F y, F z)
\end{aligned}
$$

by Step 1 .

Step 3. $d(P(F y), P(F z))<2 A$.

Proof. By hypothesis $d\left(F \circ \sigma\left[t_{0}, u_{0}\right], \sigma^{*}\right) \geqslant R+\alpha, d\left(F \sigma t_{0}, \sigma^{*}\right)=d\left(F y, \sigma^{*}\right)=R$ $+\alpha, d\left(F \sigma u_{0}, \sigma^{*}\right)=d\left(F z, \sigma^{*}\right)=R+\alpha$ and $d(y, z)=d\left(\sigma u_{0}, \sigma t_{0}\right)>r$. The assertion now follows from Lemma 2.

Finally, we complete the proof of Lemma 3 and, hence, of the proposition. We have shown that

$$
\begin{aligned}
d\left(F x, \sigma^{*}\right) & \leqslant d(F x, F y)+d\left(F y, \sigma^{*}\right) \\
& \leqslant(2 \alpha / r \zeta) d(F y, F z)+(R+\alpha) \quad(\text { by Step 2) } \\
& \leqslant(2 \alpha / r \zeta)(2 R+2 \alpha+2 A)+(R+\alpha) \quad \text { (by Step 3) }
\end{aligned}
$$

since

$$
\begin{aligned}
d(F y, F z) & \leqslant d(F y, P F y)+d(P F y, P F z)+d(P F z, F z) \\
& \leqslant(R+\alpha)+2 A+(R+\alpha) .
\end{aligned}
$$

This completes the proof.

Appendix I. We prove Proposition 2.2. Let $G, \tilde{G}$ have the properties stated. For each $\phi \in \Gamma$ and each $p \in H$ we have $\tilde{G}(\phi p)=\theta(\phi, p) \tilde{G}(p)$ for some $\theta(\phi, p) \in \Gamma^{*}$ since $\pi^{*} \circ \tilde{G}=G \circ \pi$. The element $\theta(\phi, p)$ varies continuously in $p$ and, since $\Gamma^{*}$ is a discrete group, it follows that $\theta(\phi, p) \equiv \theta(\phi)$ for all $p \in H$. It is routine to show that $\theta$ is an isomorphism. It remains to show that $\tilde{G}$ is a $(\zeta, r)$ pseudoisometry for suitable positive constants $\zeta, r$. The uniform continuity of $\tilde{G}$ is clear since $H / \Gamma$ is compact and $\tilde{G}$ is $\theta$-equivariant. We begin with the following

LEMMA. For any constant $r>0$ we can find a constant $\delta>0$ such that if $d(p, q) \geqslant \delta$, then $d(\tilde{G} p, \tilde{G} q) \geqslant r$. 
Proof. Suppose that the assertion is false for some $r>0$. Then we may choose sequences $\left\{p_{n}\right\} \subseteq H$ and $\left\{q_{n}\right\} \subseteq H$ such that $d\left(p_{n}, q_{n}\right) \geqslant n$ but $d\left(\tilde{G} p_{n}, \tilde{G} q_{n}\right)<r$ for every $n$. Since $H / \Gamma$ is compact we may choose $\left\{\phi_{n}\right\} \subseteq \Gamma$ so that $\left\{\phi_{n}\left(p_{n}\right)\right\}$ is a bounded sequence in $H$. Replacing $q_{n}$ by $\phi_{n}\left(q_{n}\right)$ and using the $\theta$-equivariance of $\tilde{G}$, then we may assume to begin with that $\left\{p_{n}\right\}$ is a bounded sequence in $H$. Let $\left\{p_{n}\right\}$ converge to a point $p \in H$ by passing to a subsequence. Then $\tilde{G}\left(p_{n}\right) \rightarrow \tilde{G}(p)$.

Choose another sequence $\left\{\Psi_{n}\right\} \subseteq \Gamma$ so that $\left\{\Psi_{n}\left(q_{n}\right)\right\}=\left\{q_{n}^{*}\right\}$ is a bounded sequence in $H$, and let $\left\{q_{n}^{*}\right\}$ converge to $q^{*}$ by passing to a further subsequence. Then $\tilde{G}\left(q_{n}^{*}\right) \rightarrow \tilde{G}\left(q^{*}\right)$ and, in particular, $\left\{\tilde{G}\left(q_{n}^{*}\right)\right\}$ is a bounded sequence in $H^{*}$. However, $\left\{\tilde{G}\left(q_{n}\right)\right\}$ is also a bounded sequence in $H^{*}$ since $\tilde{G}\left(p_{n}\right) \rightarrow \tilde{G}(p)$ and $d\left(\tilde{G} p_{n}, \tilde{G} q_{n}\right)<r$ for every $n$. Observe now that $\tilde{G}\left(q_{n}^{*}\right)=\tilde{G}\left(\Psi_{n} q_{n}\right)=\Psi_{n}^{*} \tilde{G}\left(q_{n}\right)$, where $\Psi_{n}^{*}=\theta\left(\Psi_{n}\right)$. Since $d\left(p_{n}, q_{n}\right) \rightarrow+\infty$ and $p_{n} \rightarrow p$, the sequence $\left\{\Psi_{n}\right\}$ contains infinitely many distinct elements and, hence, so does $\left\{\Psi_{n}^{*}\right\}$ since $\theta$ is an isometry. The proper discontinuity of $\Gamma^{*}$ and the boundedness of $\left\{\tilde{G}\left(q_{n}\right)\right\}$ now imply that $\Psi_{n}^{*} \tilde{G}\left(q_{n}\right)$ is an unbounded sequence, which contradicts the conclusion above and proves the lemma.

We now complete the proof of Proposition 2.2. Since $H^{*} / \Gamma^{*}$ is compact the diameter of $H^{*} / \Gamma^{*}$ is $\leqslant R$ for some constant $R>0$. Therefore for any points $p^{*}$, $q^{*}$ in $H^{*}$ we can find $\Psi^{*} \in \Gamma^{*}$ so that $d\left(\Psi^{*} p^{*}, q^{*}\right)<R$. It follows by the $\theta$-equivariance of $\tilde{G}$ that for any point $q^{*} \in H^{*}$ we can find a point $p \in H$ such that $d\left(\tilde{G} p, q^{*}\right) \leqslant R$.

By the lemma above we may choose $\delta>0$ so that if $d(p, q) \geqslant \delta$, then $d(\tilde{G} p, \tilde{G} q)$ $\geqslant 3 R$, where $R$ is the constant of the previous paragraph. We assert that if $d(p, q) \geqslant \delta$, then $d(\tilde{G} p, \tilde{G} q) \geqslant(R / \delta) d(p, q)$, which will prove the proposition.

Let $p, q$ be given with $d(p, q) \geqslant \delta$. Then $d(\tilde{G} p, \tilde{G} q) \geqslant 3 R$. Let $N \geqslant 3$ be that integer such that $N R \leqslant d(\tilde{G} p, \tilde{G} q)<(N+1) R$. Partition the geodesic segment $\gamma$ from $\tilde{G} p$ to $\tilde{G} q$ into $N+1$ equal segments of length $d(\tilde{G} p, \tilde{G} q) / N+1<R$. That is, choose points $z_{0}, z_{1}, \ldots, z_{N+1}$ on $\gamma$ so that $z_{0}=\tilde{G} p, z_{N+1}=\tilde{G} q, d(\tilde{G} p, \tilde{G} q)=$ $\sum_{i=0}^{N} d\left(z_{i}, z_{i+1}\right)$ and $d\left(z_{i}, z_{i+1}\right)=d(\tilde{G} p, \tilde{G} q) / N+1<R$ for $0 \leqslant i \leqslant N$. By the remarks above we may choose points $y_{0}, y_{1}, \ldots, y_{N+1}$ in $H$ so that $d\left(\tilde{G} y_{i}, z_{i}\right)<R$ for every $i, 0 \leqslant i \leqslant N+1$. We require further that $y_{0}=p$ and $y_{N+1}=q$.

We assert that $d\left(y_{i}, y_{i+1}\right)<\delta$ for every $i$. To do this it suffices to show that $d\left(\tilde{G} y_{i}, \tilde{G} y_{i+1}\right)<3 R$ for every $i$. If $i=0$ then

$$
d\left(\tilde{G} y_{0}, \tilde{G} y_{1}\right)=d\left(\tilde{G} p, \tilde{G} y_{1}\right) \leqslant d\left(\tilde{G} p, z_{1}\right)+d\left(z_{1}, \tilde{G} y_{1}\right) \leqslant 2 R<3 R
$$

since $\tilde{G} p=z_{0}$. If $1 \leqslant i \leqslant N-1$ then

$$
d\left(\tilde{G} y_{i}, \tilde{G} y_{i+1}\right) \leqslant d\left(\tilde{G} y_{i}, z_{i}\right)+d\left(z_{i}, z_{i+1}\right)+d\left(z_{i+1}, \tilde{G} y_{i+1}\right)<3 R .
$$

If $i=N$ then

$$
d\left(\tilde{G} y_{N}, \tilde{G} y_{N+1}\right) \leqslant d\left(\tilde{G} y_{N}, z_{N}\right)+d\left(z_{N}, z_{N+1}\right) \leqslant 2 R<3 R
$$

since $z_{N+1}=\tilde{G} q$. Therefore $d\left(y_{i}, y_{i+1}\right)<\delta$ for every $i$.

Finally

$d(p, q)=d\left(y_{0}, y_{N+1}\right) \leqslant \sum_{i=0}^{N} d\left(y_{i}, y_{i+1}\right)<N \delta=(N R)(\delta / R) \leqslant(\delta / R) d(\tilde{G} p, \tilde{G} q)$.

It follows that $d(\tilde{G} p, \tilde{G} q) \geqslant(R / \delta) d(p, q)$. 
Appendix II. Here we prove the propositions of $\$ 3$ and also contruct an example of a compact 3-manifold $M$ of nonpositive curvature that admits a free homotopy class $[\alpha]$ such that $[\alpha]$ is bounded but $[\alpha]^{n}$ is unbounded for some $n>2$. The example is also typical of what one may find in case (3) of Proposition 3.8.

Proof of Proposition 3.1. By the discussion of $\$ 3$ we may choose an element $\phi \neq 1$ in $\Gamma$ such that $T(\phi)$ is the free homotopy class in $M$ that is represented by the periodic geodesics $\gamma_{1}$ and $\gamma_{2}$. Choose lifts $\tilde{\gamma}_{1}, \tilde{\gamma}_{2}$ of $\gamma_{1}, \gamma_{2}$ such that $\tilde{\gamma}_{1}(a)=$ $\left(\phi \circ \tilde{\gamma}_{1}\right)(0)$ and $\tilde{\gamma}_{2}(b)=\left(\phi \circ \tilde{\gamma}_{2}\right)(0)$, where $a=L\left(\gamma_{1}\right)$ and $b=L\left(\gamma_{2}\right)$. The fact that $\gamma_{1}$ and $\gamma_{2}$ are smoothly closed implies that $\phi_{*} \tilde{\gamma}_{1}^{\prime}(0)=\tilde{\gamma}_{1}^{\prime}(a)$ and $\phi_{*} \tilde{\gamma}_{2}^{\prime}(0)=\tilde{\gamma}_{2}^{\prime}(b)$ and, hence, $\left(\phi \circ \tilde{\gamma}_{1}\right)(t)=\tilde{\gamma}_{1}(t+a)$ and $\left(\phi \circ \tilde{\gamma}_{2}\right)(t)=\tilde{\gamma}_{2}(t+b)$ for all $t \in \mathbf{R}$. By Proposition 4.2 of [2] we see that $a=b=$ the minimum value of the displacement function $d_{\phi}$. Hence $\gamma_{1}$ and $\gamma_{2}$ have the same length. By Proposition 6.7 of [11] the geodesics $\tilde{\gamma}_{1}, \tilde{\gamma}_{2}$ are equivalent in $H$ and bound a flat strip $S$ whose longitudinal geodesics are translated by $\phi$. More precisely, there exists a smooth, totally geodesic isometric imbedding $\tilde{F}: \mathbf{R} \times\left[0, R^{*}\right] \rightarrow H$ whose image is $S$ and such that $\phi(\tilde{F}(s, t))=\tilde{F}(s+c, t)$ for all $(s, t)$, where $c$ is the minimum value of $d_{\phi}$. The number $R^{*}$ is the Hausdorff distance between $\tilde{\gamma}_{1}$ and $\tilde{\gamma}_{2}$.

Finally if $p: \mathbf{R} \rightarrow S^{1}(c)$ and $\pi: H \rightarrow M=H / \Gamma$ are the projection maps, then define $F: S^{1}(c) \times\left[0, R^{*}\right] \rightarrow M$ by $F(p s, t)=(\pi \circ \tilde{F})(s, t)$.

Proof of Proposition 3.5. We prove the assertions in the order $(1) \Rightarrow(2) \Rightarrow$ (3) $\Rightarrow(2) \Rightarrow(1)$.

(1) $\Rightarrow(2)$ The fact that $[\alpha]=T(\phi)$ is Clifford implies that for each point $p \in H$ the geodesic $\gamma_{p}$ joining $p$ to $\phi p$ projects to a smooth periodic geodesic in [ $\alpha$ ]. Hence $\phi$ translates $\gamma_{p}$ for every $p \in H$ and by Proposition 4.2 of [2] the function $d_{\phi}$ is constant in $\boldsymbol{H}$. Therefore $\phi$ is a Clifford translation.

$(2) \Rightarrow(3)$ Let $C$ be the subgroup of Clifford translations in $\Gamma$, and let $\Gamma^{*}$ denote the centralizer of $C$ in $\Gamma$. The group $C$ acts by translations on the Euclidean de Rham factor of $H$ by Theorem 1 of [27] and hence $C$ is free abelian of rank $k \leqslant \operatorname{dim} H$. By the argument of Lemma 3 in [29] it follows that $\Gamma^{*}$ has finite index in $\Gamma$.

(3) $\Rightarrow$ (2) By hypothesis $\phi$ has only finitely many distinct conjugates $\phi_{1}, \ldots, \phi_{m}$ in $\Gamma$, where $\phi_{1}=\phi$. Let $f=\sum_{i=1}^{m}\left(d_{\phi_{1}}\right)^{2}$. Then $f$ is a $C^{\infty}$ convex function in $H$ by Proposition 4.2 of [2] and $f$ is invariant under $\Gamma$. Since $H / \Gamma$ is compact it follows that $f$ takes on all of its values on a compact subset of $H$. Therefore $f$ and, consequently, $\left(d_{\phi}\right)^{2}$ are functions bounded above in $H$. For every geodesic $\gamma$ the function $\left(d_{\phi} \circ \gamma\right)^{2}(t)$ is positive, convex and bounded above on $\mathbf{R}$. Hence $d_{\phi} \circ \gamma$ is a constant function for each geodesic $\gamma$ of $H$, which implies that $d_{\phi}$ is constant in $H$.

(2) $\Rightarrow$ (1) Since $d_{\phi}$ is a constant function in $H, \phi$ translates the geodesic $\gamma_{p}$ joining $p$ to $\phi p$ for every point $p \in H$ by Proposition 4.2 of [2]. Hence $\pi \circ \gamma_{p}$ is a smooth periodic geodesic representing $T(\phi)=[\alpha]$ for every $p \in H$.

Proof of Proposition 3.6. We prove the assertions of the proposition in the order $(1) \Rightarrow(2) \Rightarrow(3) \Rightarrow(9) \Rightarrow(7) \Rightarrow(1)$ and $(3) \Rightarrow(4) \Rightarrow(8) \Rightarrow(1)$ and $(2) \Rightarrow(5) \Rightarrow$ $(6) \Rightarrow(2)$.

(1) $\Rightarrow(2)$ Let $[\alpha]=T(\phi)$ be unbounded and let $\gamma$ be a geodesic of $H$ that is translated by $\phi$ with period $\omega$. Let $C(\phi)$ denote the centralizer of $\phi$ in $\Gamma$, and 
suppose that $C(\phi)$ is infinite cyclic with generator $\Psi$. We shall obtain a contradiction, proving (2).

Let $\gamma^{*}$ be a geodesic translated by $\Psi$. Then $\phi$ also translates $\gamma^{*}$ since $\phi$ is a power of $\Psi$. Since $[\alpha]$ is unbounded we can find a sequence of geodesics $\left\{\sigma_{n}\right\}$ in $H$ equivalent to $\gamma^{*}$ such that $\operatorname{Hd}\left(\sigma_{n}, \gamma^{*}\right) \rightarrow+\infty$ and $\phi$ translates each geodesic $\sigma_{n}$ with period $\omega>0$. Since $M=H / \Gamma$ is compact we may choose a sequence $\left\{\xi_{n}\right\} \subseteq \Gamma$ such that $\left\{\xi_{n}\left(\sigma_{n} 0\right)\right\}$ is a bounded sequence in $H$. If $\beta_{n}=\xi_{n} \circ \sigma_{n}$ and $\phi_{n}=\xi_{n} \phi \xi_{n}^{-1}$, then $\phi_{n}$ translates $\beta_{n}$ with period $\omega>0$ for every $n$. Since $\left\{\beta_{n}(0)\right\}$ is bounded it follows from the proper discontinuity of $\Gamma$ that only finitely many of the isometries $\phi_{n}$ are distinct. Passing to a subsequence we may assume that $\phi_{n}=\phi_{1}$ for every $n \geqslant 1$ and, hence, $\xi_{n}=\xi_{1} \alpha_{n}$, where $\alpha_{n} \in \Gamma$ commutes with $\phi$ for every $n$. Since $\alpha_{n} \in C(\phi)$ we may choose integers $r_{n}$ so that $\alpha_{n}=\Psi^{r_{n}}$ for every $n$.

By definition $\beta_{n}=\xi_{n} \circ \sigma_{n}=\xi_{1} \alpha_{n} \circ \sigma_{n}=\xi_{1} \Psi^{r_{n}} \circ \sigma_{n}$. Since $\Psi$ translates $\gamma^{*}$ and $\sigma_{n}$ is equivalent to $\gamma^{*}$, it follows that $\Psi^{r_{n}} \circ \sigma_{n}=\xi_{1}^{-1} \circ \beta_{n}$ is equivalent to $\gamma^{*}$ for every $n$. Moreover $\operatorname{Hd}\left(\Psi^{r_{n}} \circ \sigma_{n}, \gamma^{*}\right)$ is uniformly bounded since $\left\{\left(\xi_{1}^{-1} \circ \beta_{n}\right)(0)\right\}$ is a bounded sequence in $H$ by the way in which $\xi_{n}$ and $\beta_{n}$ were chosen. On the other hand,

$$
\operatorname{Hd}\left(\Psi^{r_{n}} \circ \sigma_{n}, \gamma^{*}\right)=\operatorname{Hd}\left(\Psi^{r_{n}} \circ \sigma_{n}, \Psi^{r_{n}} \circ \gamma^{*}\right)=\operatorname{Hd}\left(\sigma_{n}, \gamma^{*}\right) \rightarrow+\infty
$$

by the way in which the geodesics $\sigma_{n}$ were chosen. This contradiction proves that $C(\phi)$ cannot be infinite cyclic and proves (2).

(2) $\Rightarrow(3)$ Let $\gamma$ be a geodesic translated by $\phi$. Let $C(\phi)$ denote the centralizer of $\phi$ in $\Gamma$ and suppose that $C(\phi)$ is not infinite cyclic. Then there exists $\Psi \neq 1$ in $C(\phi)$ such that $\Psi$ translates a geodesic not equivalent to $\gamma$, for otherwise $C(\phi)$ would be infinite cyclic by Theorem 6.11 of [11]. Now let $G$ be the subgroup of $\Gamma$ generated by $\phi$ and $\Psi$. Clearly $C$ is abelian and $G$ has no elements of finite order since any elements of finite order would have a fixed point in $H$ by a theorem of E. Cartan. The group $G$ cannot be infinite cyclic with generator $\xi$; in this case both $\Psi$ and $\phi$ would translate any geodesic $\gamma^{*}$ that $\xi$ translates, and such a geodesic $\gamma^{*}$ would be equivalent to $\gamma$ by Proposition 6.7 of [11], contradicting the choice of $\Psi$. Therefore $G$ is a free abelian group of rank 2 .

(3) $\Rightarrow$ (9) Let $\Psi \in \Gamma$ be an element such that $\phi$ and $\Psi$ generate a free abelian group $G \subseteq \Gamma$ of rank 2. By Theorem 1 of [19] there exists a totally geodesic isometric imbedding $\tilde{F}: \mathbf{R}^{2} \rightarrow H$ such that $G$ leaves $\tilde{F}\left(\mathbf{R}^{2}\right)$ invariant and operates by translations on $\tilde{F}\left(\mathbf{R}^{2}\right)$ so that $\tilde{F}\left(\mathbf{R}^{2}\right) / G$ is compact (hence a 2-torus). More precisely, there exist linearly independent vectors $\xi_{1}, \xi_{2} \in \mathbf{R}^{2}$ such that $(\phi \circ \tilde{F})(s, t)=$ $\tilde{F}\left(\xi_{1}+(s, t)\right)$ and $(\Psi \circ \tilde{F})(s, t)=\tilde{F}\left(\xi_{2}+(s, t)\right)$ for all $(s, t) \in \mathbf{R}^{2}$. If $\tilde{G}$ denotes the group of translations of $\mathbf{R}^{2}$ generated by $\xi_{1}, \xi_{2}$ and if $T^{2}$ is the quotient $\mathbf{R}^{2} / \tilde{G}$, then there is a totally geodesic, isometric immersion $F: T^{2} \rightarrow M$ such that $F \circ p=$ $\pi \circ \tilde{F}$, where $p: \mathbf{R}^{2} \rightarrow T^{2}$ and $\pi: H \rightarrow M$ are projection maps.

(9) $\Rightarrow$ (7) Let $F: T^{2} \rightarrow M$ satisfy the hypotheses of (7). Express $T^{2}$ as a quotient $\mathbf{R}^{2} / G$ with projection $p: \mathbf{R}^{2} \rightarrow T^{2}$, where $G$ is a translation group generated by linearly independent vectors $\xi_{1}, \xi_{2}$. Without loss of generality we may interpret the hypothesis on $F$ to mean that the curves $u \rightarrow(F \circ p)\left[(s, t)+u \xi_{1}\right]$ are smooth periodic geodesics belonging to $[\alpha]$ for every $(s, t) \in \mathbf{R}^{2}$. We may lift $F$ to obtain a 
totally geodesic isometric immersion $\tilde{F}: \mathbf{R}^{2} \rightarrow H$ such that $\pi \circ \tilde{F}=F \circ p$, where $\pi$ : $H \rightarrow M=H / \Gamma$ is the covering projection. The map $\tilde{F}$ must actually be an imbedding since there is a unique geodesic in $H$ joining any two distinct points of $H$. For each $(s, t) \in \mathbf{R}^{2}$ the geodesic $u \rightarrow \tilde{F}\left[(s, t)+u \xi_{1}\right]$ projects under $\pi$ to a smooth periodic geodesic in $[\alpha]$, and hence the discreteness of $\Gamma$ implies that there exists an element $\tilde{\phi} \neq 1$ in $\Gamma$ with $T(\tilde{\phi})=[\alpha]$ that translates each such geodesic. Since $T(\tilde{\phi})=T(\phi)=[\alpha]$ it follows that $\tilde{\phi}$ is conjugate to $\phi$. To prove that $\phi$ fixes at least 3 points in $H(\infty)$ it therefore suffices to prove that $\tilde{\phi}$ fixes at least 3 points in $H(\infty)$.

The preceding paragraph implies that $\tilde{\phi}$ translates the maximal geodesic from $q$ to $\tilde{\phi} q$ for each point $q \in \tilde{F}\left(\mathbf{R}^{2}\right)$. In particular, $d_{\tilde{\phi}}: q \rightarrow d(q, \tilde{\phi} q)$ assumes its minimal value $\omega>0$ at each point of $\tilde{F}\left(\mathbf{R}^{2}\right)$. Fix a point $p \in \tilde{F}\left(\mathbf{R}^{2}\right)$, and let $\sigma$ be any geodesic ray starting at $p$ and contained in $\tilde{F}\left(\mathbf{R}^{2}\right)$. Then $d_{\tilde{\phi}}(\sigma t) \equiv \omega$ for all $t \in \mathbf{R}$ and, in particular, $\tilde{\phi}$ fixes $\sigma(\infty)$. Since $\sigma$ is arbitrary $\tilde{\phi}$ fixes an infinite number of points in $H(\infty)$.

(7) $\Rightarrow$ (1) Let $[\alpha]=T(\phi)$, and let $\gamma$ be a geodesic translated by $\phi$ with period $\omega>0$. By hypothesis there exists a point $z \in H(\infty)$ that is fixed by $\phi$ and distinct from $\gamma(\infty)$ and $\gamma(-\infty)$. Let $\sigma$ be the unit speed geodesic ray with $\sigma(0)=\gamma(0)$ and $\sigma(\infty)=z$. The displacement function $d_{\phi}$ assumes its minimum value $\omega$ at all points of $\gamma$, in particular, at $\sigma(0)$. The geodesics $\sigma$ and $\phi \circ \sigma$ are asymptotic since $\phi$ fixes $z=\sigma(\infty)$ and, hence, $t \rightarrow\left(d_{\phi} \circ \sigma\right)^{2}(t)$ is bounded above for $t>0$. The function $\left(d_{\phi} \circ \sigma\right)^{2}$ is $C^{\infty}$, convex on $\mathbf{R}$ and bounded above for $t \geqslant 0$ and, hence, $\left(d_{\phi} \circ \sigma\right)^{2}$ is nonincreasing on $\mathbf{R}$. Therefore $d_{\phi}(\sigma t) \leqslant d_{\phi}(\sigma 0)=\omega$ for all $t>0$, but $\omega$ is the minimum value of $d_{\phi}$ and, hence, $d_{\phi}(\sigma t) \equiv \omega$ for $t>0$. Therefore $\phi$ translates the geodesic $\gamma_{t}$ from $\sigma(t)$ to $\phi \sigma(t)$ for every $t \geqslant 0$. It follows that [ $\alpha$ ] is unbounded since $\operatorname{Hd}\left(\gamma_{t}, \gamma\right) \rightarrow+\infty$ as $t \rightarrow+\infty$.

(3) $\Rightarrow$ (4) This follows immediately from Theorem 1 of [19].

$(8) \Rightarrow(1)$ is obvious. We show that

(4) $\Rightarrow(8)$ Let $\tilde{F}: \mathbf{R}^{2} \rightarrow H$ be as in the statement of (4). Rotating the axes in $\mathbf{R}^{2}$ if necessary we may assume that $(\phi \circ \tilde{F})(s, t)=\tilde{F}(s+\omega, t)$ for all $(s, t)$ in $\mathbf{R}^{2}$. If $S^{1}$ denotes here the circle of length $\omega=\inf d_{\phi}$, then let $p: \mathbf{R}^{1} \rightarrow S^{1}$ denote the covering map $t \rightarrow \exp (2 \pi i t / \omega)$. If we define $F: S^{1} \times \mathbf{R} \rightarrow M$ by $F[(p \times 1)(s, t)]=$ $(\pi \circ \tilde{F})(s, t)$ for $(s, t) \in \mathbf{R} \times \mathbf{R}$, then $F$ is well defined and satisfies the properties of (8).

$(2) \Rightarrow(5)$ Since $[\alpha]$ is unbounded it must have by definition more than one periodic geodesic representative. By the equivalence of (2) and (3) we may choose an element $\psi \in \Gamma$ such that $\phi$ and $\psi$ generate a free abelian group $G \subseteq \Gamma$ of rank 2 . If $\gamma$ is any geodesic translated by $\phi$, then $\gamma$ and $\psi^{n} \circ \gamma$ are equivalent geodesics whose Hausdorff distance diverges to $+\infty$ as $n \rightarrow+\infty$ by the fact that $\phi$ and $\psi$ operate as independent translations on some flat plane in $H$; see the proof of (3) $\Rightarrow$ (9) for details.

Now let $\gamma_{0}, \gamma_{1}$ be any periodic geodesics that belong to [ $\alpha$, and let $\tilde{\gamma}_{0}, \tilde{\gamma}_{1}$ be lifts to $H$ that are translated by $\phi$. For any integer $n \geqslant 1$ the geodesic $\psi^{n} \circ \tilde{\gamma}_{1}$ is translated by $\phi$ since $\psi^{n} \in C(\phi)$ and, moreover, $R_{n}=\operatorname{Hd}\left(\psi^{n} \tilde{\gamma}_{1}, \tilde{\gamma}_{0}\right) \rightarrow+\infty$ as 
$n \rightarrow \infty$ by the remarks above. Define a smooth, totally geodesic, isometric imbed$\operatorname{ding} \tilde{F}_{n}: \mathbf{R} \times\left[0, R_{n}\right] \rightarrow H$ such that $\tilde{F}_{n}(t, 0)=\tilde{\gamma}_{0}(t)$ and $\tilde{F}_{n}\left(t, R_{n}\right)$ parametrizes $\psi^{n} \circ \tilde{\gamma}_{1}$. Proceeding as in the proof of Proposition 3.1 we define $F_{n}: S^{1}(c) \times\left[0, R_{n}\right]$ $\rightarrow M$ by $F_{n}(p s, t)=\left(\pi \circ \tilde{F}_{n}\right)(s, t)$, where $c=\inf d_{\phi}=|[\alpha]|$ and $p: \mathbf{R} \rightarrow S^{1}(c)$ and $\pi: H \rightarrow M$ are the projection maps. Clearly the flat homotopy $F_{n}$ joins $\gamma_{0}$ to $\gamma_{1}$ for every $n$, and there are infinitely many distinct homotopies $F_{n}$ since $R_{n} \rightarrow+\infty$ as $n \rightarrow \infty$.

The result $(5) \Rightarrow(6)$ is obvious so it remains only to prove that $(6) \Rightarrow(2)$ to complete the proof of Proposition 3.6. It suffices to prove the following

LEMMA. Let $C(\phi)$ be an infinite cyclic subgroup of $\Gamma$ and let $\xi$ be the generator of $C(\phi)$ such that $\phi=\xi^{r}$ for some positive integer $r$. Let $\gamma_{0}, \gamma_{1}$ be any distinct periodic geodesics in $[\alpha]=T(\phi)$. Then there are at most $r$ distinct flat homotopies that join $\gamma_{0}$ to $\gamma_{1}$.

Proof. Let $\gamma_{0}, \gamma_{1}$ be distinct periodic geodesics that belong to [ $\alpha$ ]. We first construct flat homotopies $F_{1}, \ldots, F_{r}$, possibly not all distinct, that join $\gamma_{0}$ to $\gamma_{1}$. Then we show that any flat homotopy that joins $\gamma_{0}$ to $\gamma_{1}$ must equal $F_{j}$ for some $1 \leqslant j \leqslant r$.

For the rest of the proof let $c=\inf d_{\phi}=|[\alpha]|$ and let $p: \mathbf{R} \rightarrow S^{1}(c)$ and $\pi$ : $H \rightarrow M$ denote the covering projections. Choose geodesics $\tilde{\gamma}_{i}, i=0$, 1 , so that $\pi \circ \tilde{\gamma}_{i}=\gamma_{i}$ and $\left(\phi \circ \tilde{\gamma}_{i}\right)(t)=\tilde{\gamma}_{i}(t+c)$ for all $t$. Furthermore we may parametrize $\tilde{\gamma}_{1}$ so that $\tilde{\gamma}_{1}(0)$ is the point on $\tilde{\gamma}_{1}$ closest to $\tilde{\gamma}_{0}(0)$. For $2 \leqslant j \leqslant r$ we define $\tilde{\gamma}_{j}$ to be the geodesic $\xi^{j-1}\left(\tilde{\gamma}_{1}\right)$ and parametrize $\tilde{\gamma}_{j}$ so that $\tilde{\gamma}_{j}(0)$ is the point on $\tilde{\gamma}_{j}$ closest to $\tilde{\gamma}_{0}(0)$. The geodesics $\tilde{\gamma}_{j}$ are equivalent to $\tilde{\gamma}_{0}$ for $1 \leqslant j \leqslant r$ since $\phi$ translates $\tilde{\gamma}_{j}$, and by the choice of parametrization there exists a smooth, totally geodesic, isometric imbed$\operatorname{ding} \tilde{F}_{j}: \mathbf{R} \times\left[0, R_{j}\right] \rightarrow H$ such that $\tilde{F}_{j}(t, 0)=\tilde{\gamma}_{0}(t)$ and $\tilde{F}_{j}\left(t, R_{j}\right)=\tilde{\gamma}_{j}(t)$ for all $t$ and all $j$. The number $R_{j}$ is the Hausdorff distance between $\tilde{\gamma}_{0}$ and $\tilde{\gamma}_{j}$. We now define $F_{j}: S^{1}(c) \times\left[0, R_{j}\right] \rightarrow M$ by $F_{j}(p t, s)=\left(\pi \circ \tilde{F}_{j}\right)(t, s)$ for all $t, s$. Clearly each $F_{j}$ is a flat homotopy that joins $\gamma_{0}$ to $\gamma_{1}$. We let $F_{j}$ denote also the translated homotopy $(p t, s) \rightarrow\left(\pi \circ \tilde{F}_{j}\right)(t+c, s)$, where $c \in \mathbf{R}$ is arbitrary.

Now let $F: S^{1}(c) \times[0, R] \rightarrow M$ be any smooth, totally geodesic, isometric immersion such that $F(p t, 0)=\gamma_{0}(t)$ and $F\left(S^{1} \times\{R\}\right)=\gamma_{1}$ where $\gamma_{0}$ has the parametrization induced from $\tilde{\gamma}_{0}$. Lift the homotopy $F$ to obtain a smooth, totally geodesic isometric imbedding $\tilde{F}: \mathbf{R} \times[0, R] \rightarrow H$ such that $(\pi \circ \tilde{F})(t, s)=F(p t, s)$ for all $s, t$. Replacing $\tilde{F}$ by $\psi \circ \tilde{F}$ for some $\psi \in \Gamma$ we may assume that $\tilde{F}(t, 0)=$ $\tilde{\gamma}_{0}(t)$ for all $t \in \mathbf{R}$. Let $\sigma(t)=\tilde{F}(t, R)$. To show that $F=F_{j}$ for some $j$ it suffices to show that $\tilde{F}=\tilde{F}_{j}$ or, equivalently, that $\sigma(t)=\tilde{\gamma}_{j}(t)$ for all $t$.

Observe that $s \rightarrow \tilde{F}(0, s), 0 \leqslant s \leqslant R$, is a geodesic segment in $H$ that joins $\tilde{\gamma}_{0}(0)$ to $\sigma(0)$ and is mutually perpendicular to both $\tilde{\gamma}_{0}$ and $\sigma$. It now suffices to show that $\sigma=\tilde{\gamma}_{j}$ as a point set, for then the parametrizations of $\sigma$ and $\tilde{\gamma}_{j}$ will automatically agree. The geodesic $\sigma(t)$ is a lift of $\gamma_{1}$ and, hence, $\sigma(t)=\left(\psi \circ \tilde{\gamma}_{1}\right)(t+a)$ for some $\psi \in \Gamma, a \in \mathbf{R}$ and all $t$. Since $d\left(\sigma t, \tilde{\gamma}_{0} t\right) \equiv R$ it follows that $\psi$ fixes both $x=\tilde{\gamma}_{0}(\infty)$ $=\tilde{\gamma}_{1}(\infty)$ and $y=\tilde{\gamma}_{0}(-\infty)=\tilde{\gamma}_{1}(-\infty)$. Hence the element $\rho=[\psi, \phi]=\psi \phi \psi^{-1} \phi^{-1}$ fixes both $x$ and $y$ and leaves invariant all horospheres at $x$ and $y$ by Proposition 
6.6 of [11] and the discussion preceding it. Note that the isometry $\phi$ translates the geodesic $s \rightarrow \tilde{F}(s, t)$ for every $t \in[0, R]$ since it translates the geodesic $\tilde{\gamma}_{0}(s)=$ $\tilde{F}(s, 0)$ and $(\pi \circ \tilde{F})(0, t)=(\pi \circ \tilde{F})(c, t)$ for all $t \in[0, R]$. In particular, $\phi$ translates $\sigma(s)=\left(\psi \circ \tilde{\gamma}_{1}\right)(s+a)=\tilde{F}(s, R)$. It follows that $\rho=[\psi, \phi]$ leaves $\sigma$ invariant as a point set. Since $\sigma$ meets a given horosphere at $x$ or $y$ in a single point we conclude that $\rho$ fixes every point of $\sigma$ and hence $\rho=1$ since $\Gamma$ contains no elliptic elements. Therefore $\psi \in C(\phi)$ and we may write $\psi=\xi^{m}=\xi^{j-1} \phi^{k}$ for integers $m, j, k$ where $m=k r+(j-1)$ and $2 \leqslant j \leqslant r$. Finally $\sigma=\psi \tilde{\gamma}_{1}=\xi^{m} \tilde{\gamma}_{1}=\xi^{j-1} \tilde{\gamma}_{1}=\tilde{\gamma}_{j}$, which completes the proof of the lemma and also of $(6) \Rightarrow(2)$.

Proof of Proposition 3.7. Express $M$ as a quotient $H / \Gamma$ and let $C^{*}$ denote the subgroup of $\Gamma$ consisting of Clifford translations of $H$. By hypothesis and Proposition $3.5, C^{*} \neq\{1\}$. Since Clifford translations act as ordinary translations on the Euclidean de Rham factor of $H$ it follows that $C^{*}$ is free abelian of rank $k \leqslant \operatorname{dim} H$. If $\Gamma^{*}$ denotes the centralizer of $C^{*}$ in $\Gamma$, then $\Gamma^{*}$ has finite index in $\Gamma$ by the argument of Lemma 3 of [29].

Let $[\alpha]$ be a given free homotopy class in $M$ and choose $\phi \in \Gamma$ so that $[\alpha]=T(\phi)$. Let $\gamma$ be a geodesic translated by $\phi$. For each integer $n>1$ let $C\left(\phi^{n}\right)$ denote the centralizer of $\phi^{n}$ in $\Gamma$. We consider two cases: (1) For some $n \geqslant 1$ there exists $\Psi \neq 1$ in $C\left(\phi^{n}\right)$ such that $\Psi$ translates a geodesic not equivalent to $\gamma$. (2) For any $n \geqslant 1$ and any $\Psi \neq 1$ in $C\left(\phi^{n}\right)$ there exists a geodesic $\gamma^{*}$ equivalent to $\gamma$ that is translated by $\Psi$.

In the first case let $G$ be the subgroup of $\Gamma$ generated by $\phi^{n}$ and $\Psi$. Then $G$ is free abelian of rank 2 by the argument used in the proof of $(2) \Rightarrow(3)$ in Proposition 3.6. It follows that $[\alpha]^{n}=T\left(\phi^{n}\right)$ is unbounded by Proposition 3.6.

Suppose that case (2) holds and let $G$ be the set union of the groups $C\left(\phi^{n}\right)$ for all $n \geqslant 1$. If $\xi, \Psi$ are elements of $G$ then $\xi \in C\left(\phi^{m}\right)$ and $\Psi \in C\left(\phi^{n}\right)$ for suitable integers $m, n \geqslant 1$. Hence both $\xi$ and $\Psi$ lie in $C\left(\phi^{m n}\right)$. This observation shows that $G$ is a group. By the hypothesis of case (2) every element $\Psi \neq 1$ in $G$ translates some geodesic equivalent to $\gamma$. Hence $G$ is infinite cyclic with generator $\xi$ by Theorem 6.11 of [11].

Since $\Gamma^{*}$ has finite index in $\Gamma$ we may choose an integer $n \geqslant 1$ such that $\phi^{n} \in \Gamma^{*}$. Then $C^{*}$, the subgroup of Clifford translations of $\Gamma$, is contained in $C\left(\phi^{n}\right)$ and hence in $G$ since $\Gamma^{*}$ centralizes $C^{*}$. It follows that we may choose integers $r$ and $s$ such that $\xi^{r}$ is a Clifford translation $\neq 1$ and $\xi^{s}=\phi$. Therefore $\phi^{r}=\left(\xi^{r}\right)^{s}$ is a Clifford translation $\neq 1$ and, hence, $[\alpha]^{r}=T\left(\phi^{r}\right)$ is unbounded since Clifford translations translate a geodesic through every point of $H$. This proves Proposition 3.7.

A 3-dimensional example. We construct a compact 3-manifold $M$ that admits a free homotopy class $[\alpha]$ such that $[\alpha]$ is bounded but $[\alpha]^{n}$ is unbounded for some integer $n \geqslant 2$. In fact, $[\alpha]^{n}$ is Clifford for some integer $n \geqslant 2$. This example is an illustration of case (3) of Proposition 3.8 below.

We now begin the construction. Fix an integer $g \geqslant 2$ and let $H^{*}, \Gamma^{*}$ denote, respectively, the hyperbolic plane with its $K=-1$ metric and a uniform lattice $\Gamma^{*}$ in $H^{*}$ such that $H^{*} / \Gamma^{*}$ is a compact surface of genus $g$. From the Teichmüller 
space for surfaces of genus $g$ we may choose the lattice $\Gamma^{*}$ so that $N\left(\Gamma^{*}\right)$, the normalizer of $\Gamma^{*}$ in $I\left(H^{*}\right)$, contains an elliptic orientation preserving $\beta \neq 1$. Clearly $\beta \in N\left(\Gamma^{*}\right)-\Gamma^{*}$ since $H^{*} / \Gamma^{*}$ is a smooth manifold. It is known that $\Gamma^{*}$ has finite index in $N\left(\Gamma^{*}\right)$ and, hence, $N\left(\Gamma^{*}\right)$ is a discrete subgroup of $I\left(H^{*}\right)$. Let $G^{*}$ denote the discrete subgroup of $N\left(\Gamma^{*}\right)$ generated by $\Gamma^{*}$ and $\beta$.

Let $H$ denote the Riemannian product of $\mathbf{R}$ and $H^{*}$, and let $\Gamma$ denote the direct product $\mathbf{Z} \times \Gamma^{*}$, where $\mathbf{Z}$ denotes the integers. Let $\Gamma$ act on $H$ by $(n \times \gamma)(t, p)=$ $(t+n, \gamma p)$ for every $n \times \gamma \in \mathbf{Z} \times \Gamma^{*}$ and $(t, p) \in \mathbf{R} \times H^{*}$. Let $\beta \in N\left(\Gamma^{*}\right)$ be the elliptic element chosen above, and let $\beta$ act on $H$ by $\beta(t, p)=(t+1 / N, \beta p)$, where $N$ is the order of $\beta$ in $G^{*}$. Now let $G$ be the group of isometries of $H$ generated by $\beta$ and $\Gamma=\mathbf{Z} \times \Gamma^{*}$.

We assert that $M=H / G$ is the desired compact 3-manifold. A typical element of $G$ has the form

$$
\sigma=\beta^{k_{1}} \gamma_{1} \beta^{k_{2}} \gamma_{2} \cdots \beta^{k_{r}} \gamma_{r}
$$

where $k_{i}$ is an integer and $\gamma_{i}=n_{i} \times \sigma_{i}$ lies in $\mathbf{Z} \times \Gamma^{*}$ for $1 \leqslant i \leqslant r$. Since $\beta$ normalizes $\Gamma^{*}$ in $G^{*}$ it is easy to see that

$$
\sigma(t, p)=\left(t+n+(k / N), \beta^{k} \sigma^{*} p\right)
$$

for all $(t, p) \in H$, where $n=\sum n_{i}, k=\sum k_{i}$ and $\sigma^{*}$ is some element of $\Gamma^{*}$. The group $G$ is discrete since $G^{*}$ is a discrete group of isometries of $H^{*}$ and $\mathbf{Z}$ is the center of $G$. Moreover, $H / G$ is compact since $H^{*} / G^{*}$ is compact. To show that $M=H / G$ is a smooth manifold it remains only to show that $G$ contains no elliptic elements. Suppose that $\sigma \in G$ is elliptic with fixed point $(t, p)$. From the formula above for $\sigma(t, p)$, it follows that $n+(k / N)=0$. Hence $k$ is an integer multiple of $N$ and $\beta^{k}=1$. Therefore, $\sigma^{*}=\beta^{k} \sigma^{*}$ fixes $p$ since $\sigma$ fixes $(t, p)$ and it follows that $\sigma^{*}=1$ since $\Gamma^{*}$ contains no nonidentity elliptic elements. Finally, $\sigma$ must be the identity.

We have shown that $M=H / G$ is a smooth compact 3-manifold. Consider $\beta \in G$ acting on $H$ in the way described above. Let $p \in H^{*}$ be the unique fixed point of $\beta$ considered as an isometry of $H^{*}$. If $\tilde{\alpha}: \mathbf{R} \rightarrow H$ is the geodesic $t \rightarrow(t, p)$, then $(\beta \circ \tilde{\alpha})(t)=\tilde{\alpha}(t+1 / N)$ for all $t$ and $\tilde{\alpha}$ is the unique geodesic in $H$ translated by $\beta$. It follows from Proposition 3.6 that $[\alpha]=T(\beta)$ is bounded, where $\alpha=\pi \circ \tilde{\alpha}$ and $\pi: H \rightarrow M=H / G$ is the covering projection. On the other hand, $\beta^{N}(t, q)=$ $(t+1, q)$ for all $(t, q) \in H$ and hence $\beta^{N}$ is a Clifford translation of $H$. It follows that $[\alpha]^{N}=T\left(\beta^{N}\right)$ is Clifford, hence unbounded. This completes the construction of the example.

The manifold $M$ has a finite cover that splits as a Riemannian product $S^{1} \times M_{g}$, where $M_{g}=H^{*} / \Gamma^{*}$ is a surface of genus $g$. We do not know if it is possible to construct a 3-manifold $M$ that (1) has a bounded free homotopy class [ $\alpha$ ] such that $[\alpha]^{n}$ is unbounded for some integer $n$ and (2) has no finite cover that splits as a Riemannian product $S^{1} \times M_{g}$.

Proof of Proposition 3.8. This result relies heavily on results of $\S 5$ of [1]. For the convenience of the reader we state the two results of that section that are relevant here. 
SATZ 5.2. Let $M=H / \Gamma$ be a compact 3-manifold of nonpositive sectional curvature, and let $\phi \in \Gamma$ translate a geodesic $\gamma$ in $H$. Then $\gamma$ bounds a flat half-plane in $H$ if and only if some power of $\phi$ is contained in a rank 2 free abelian subgroup of $\Gamma$.

SATz 5.3. Let $M=H / \Gamma$ be a compact 3-manifold of nonpositive sectional curvature. Then $H$ admits a Euclidean de Rham factor of positive dimension if and only if every geodesic of $H$ that is translated by some nonidentity element of $\Gamma$ bounds a flat half-plane in $H$.

We now proceed to the proof of Proposition 3.8. The three cases are clearly mutually exclusive. If $M=H / \Gamma$ is flat then by the Bieberbach theorems [28, p. 100] there exists a normal abelian subgroup $\Gamma^{*}$ of finite index $n$ such that $\Gamma^{*}$ consists of Clifford translations of $H$ and $H / \Gamma^{*}$ is a flat torus. It follows immediately from Proposition 3.5 that $[\alpha]^{n}$ is Clifford for every free homotopy class $[\alpha]$ in $M$.

Next suppose that $M$ has rank 1, which by Satz 2.7 of [1] is equivalent to requiring that $M$ admit a rank 1 free homotopy class $[\alpha]$. Clearly if $[\alpha]$ is any rank 1 free homotopy class, then $[\alpha]^{n}$ is bounded for every integer $n \geqslant 1$ by the definitions of bounded and rank 1 . Conversely suppose that $[\alpha]$ is a free homotopy class such that $[\alpha]^{n}$ is bounded for every integer $n \geqslant 1$. Write $[\alpha]=T(\phi)$ for a suitable $\phi \in \Gamma$. If $[\alpha]$ did not have rank 1 , then by Satz 5.2 of [1] we could find an integer $n \geqslant 1$ and an element $\Psi \neq 1$ in $\Gamma$ such that $\phi^{n}$ and $\Psi$ generate a free abelian group of rank 2. Hence $[\alpha]^{n}=T\left(\phi^{n}\right)$ would be an unbounded free homotopy class by Proposition 3.6, contradicting our hypothesis on $[\alpha]$. Therefore $[\alpha]$ has rank 1 if $[\alpha]^{n}$ is bounded for every integer $n \geqslant 1$.

Finally we suppose that $M$ is not flat and that no free homotopy class $[\alpha]$ has rank 1. Then by Satz 5.3 of [1] (stated above) it follows that $H$ can be written as a Riemannian product $H_{1} \times H_{2}$, where $H_{1}=\mathrm{R}$ and $H_{2}$ is not flat. Since every isometry of $H$ preserves the flat de Rham factor we may write $\phi=\phi_{1} \times \phi_{2}$ for any isometry $\phi$ of $H$, where $\phi_{1}$ is an isometry of $H_{1}=\mathbf{R}$ and $\phi_{2}$ is an isometry of $H_{2}$. If $M=H / \Gamma$ let $\Gamma_{i} \subseteq I\left(H_{i}\right)$ be the set of $\phi_{i}$ for $\phi \in \Gamma, i=1,2$.

The set $H_{1}(\infty) \subseteq H(\infty)$ consists of two points and $H_{1}(\infty)$ is left invariant by $\Gamma$ by the discussion of the previous paragraph. Hence $\Gamma$ contains Clifford translations by Theorem 5.1 of [9] and part (a) of case (3) of Proposition 3.8 now follows from Propositions 3.7 and 3.5. The subgroup $C$ of Clifford translations of $\Gamma$ acts discretely on the 1-dimensional factor $H_{1}=\mathbf{R}$, and hence $C$ is infinite cyclic with generator $\Psi$. If $[\beta]=T(\Psi)$, then it follows immediately that $[\beta]$ and its powers are the Clifford free homotopy classes of $M$. This proves the first half of (b) of case (3). To establish the second half of (b) of case (3), we observe that $\Gamma_{2}$ is discrete by Theorem 5.1 of [9] and satisfies the duality condition in $H_{2}$ by the discussion on $\mathrm{p}$. 80 of [5]; see p. 78 of [5] for a definition of the duality condition. In particular, $L\left(\Gamma_{2}\right)=H_{2}(\infty)$ (the limit set of $\Gamma_{2}$; cf. [11]) since $\Gamma_{2}$ satisfies the duality condition. By Proposition 2.5 of [10], $\mathrm{H}_{2}$ satisfies the Visibility axiom, and by Proposition 8.6 of [11], $\Gamma_{2}$ contains a hyperbolic element $\phi_{2}$. Choose a corresponding $\phi=\phi_{1} \times \phi_{2}$ in $\Gamma$ and let $[\alpha]=T(\phi)$ in $M$. Since $\phi_{2}^{n}$ is never the identity for any integer $n>1$, it 
follows that $\phi^{n}$ is never a Clifford translation and $[\alpha]^{n}$ is never a Clifford free homotopy class for any integer $n \geqslant 1$. This completes (b) of case (3).

To establish (c) of case (3), we consider $\Gamma^{*}$, the subgroup of $\Gamma$ of index $\leqslant 2$ that fixes each point of the two point set $H_{1}(\infty)$. Since $H / \Gamma^{*}$ is compact $\Gamma^{*}$ satisfies the duality condition [5, p. 78] and, hence, $\Gamma_{1}^{*}$ consists of translations of $H_{1}=\mathbf{R}$ by Theorem 4.2 of [5]. By (1) and (5) of Theorem 5.1 of [9] it follows that $\Gamma^{*}$ contains Clifford translations, which operate by translations on the factor $H_{1}$ and by the identity on $H_{2}$. Since $\Gamma_{1}^{*}$ consists of translations of $H_{1}$ and since translations commute, it follows that every Clifford translation of $\Gamma^{*}$ lies in the center of $\Gamma^{*}$. (The converse assertion is also true.) Therefore the fundamental group of $M^{*}=$ $H / \Gamma^{*}$, which is isomorphic to $\Gamma^{*}$, has nontrivial center. The remaining assertion in (c) of part (3) is a consequence of the fact that $\mathrm{H}_{2}$ is not flat and of the following result of $[6]$.

THEOREM. Let $M$ be a compact manifold of nonpositive sectional curvature such that $\pi_{1}(M)$ has nontrivial center. Then $M$ admits a finite covering $M^{*}$ such that any finite covering $\bar{M}$ of $M^{*}$ is diffeomorphic (but not necessarily isometric) to a product $T^{k} \times \tilde{M}$, where $T^{k}$ is a torus with $k \geqslant 1$ and $\tilde{M}$ is a compact orientable manifold of nonpositive sectional curvature whose fundamental group has trivial center.

Proof of Proposition 3.9. Since $H$ is a nontrivial Riemannian product the discussion in [5, p. 82] shows that no free homotopy class $[\alpha]$ in $M=H / \Gamma$ has rank 1 . This proves part (1).

To prove part (2) we consider $\Gamma^{*}$, the subgroup of $\Gamma$ of index $\leqslant 2$ that leaves invariant the two foliations of $H$ tangent to the hyperbolic plane factors. Every element $\gamma^{*} \in \Gamma^{*}$ can be written $\gamma^{*}=\gamma_{1}^{*} \times \gamma_{2}^{*}$, where $\gamma_{i}^{*} \in I\left(H_{i}\right)$ for $i=1,2$ (let $H_{1}=H_{2}$ be the hyperbolic plane with curvature $\left.K \equiv-1\right)$. The fact that $\Gamma$ hence $\Gamma^{*}$, is irreducible means that $I_{0}\left(H_{i}\right) \subseteq C l\left(\Gamma_{i}^{*}\right)$ for $i=1,2$. Moreover, $\gamma_{i}^{*}$ is never the identity in $I\left(H_{i}\right)$ for $i=1,2$ if $\gamma^{*}$ is not the identity in $\Gamma^{*}$. See for example [23, pp. 86-87] for further details.

We show first that $\Gamma_{i}^{*}$ contains nonidentity elliptic elements for $i=1,2$, and for convenience we consider only $\Gamma_{2}^{*}$. Assuming that this has been done we choose $\phi=\phi_{1} \times \phi_{2} \in \Gamma^{*}$ such that $\phi_{2}$ is elliptic. Then $\phi_{2}$ has a unique fixed point $p \in H_{2}$, for otherwise $\left(\phi_{2}\right)^{2}$ would be the identity, contradicting the irreducibility of $\Gamma^{*}$ by the discussion of the previous paragraph. Since every element of $\Gamma^{*}$ is hyperbolic ( $H / \Gamma^{*}$ is compact) it follows that $\phi_{1}$ is hyperbolic in $H_{1}$ and translates a unique unit speed geodesic $\gamma_{1}(t)$. If $\gamma(t)=\left(\gamma_{1}(t), p\right)$, then $\gamma$ is the unique unit speed geodesic of $H$ translated by $\phi$ since $\phi_{2}$ is elliptic with fixed point $p$. This will imply that $[\alpha]=T(\phi)$ is bounded. For any integer $n \geqslant 1$ the element $\phi_{2}^{n}$ is not the identity and is elliptic with fixed point $p$ by the irreducibility of $\Gamma^{*}$. Therefore $\gamma$ is the unique geodesic translated by $\phi^{n}$ for any integer $n>1$ and, hence, $[\alpha]^{n}=$ $T\left(\phi^{n}\right)$ is bounded. This will prove (2).

We now show that $\Gamma_{2}^{*}$ contains nonidentity elliptic elements. Let $\beta \in I_{0}\left(H_{2}\right)$ be a nonidentity orientation preserving elliptic element. By the irreducibility of $\Gamma^{*}$ as discussed earlier we can choose a sequence $\left\{\beta_{n}\right\} \subseteq \Gamma_{2}^{*}$ that converges in $I\left(H_{2}\right)$ to 
$\beta$. We assert that $\beta_{n}$ has no fixed point in $H_{2}(\infty)$ for sufficiently large $n$ and hence must be elliptic. If this were not the case then by passing to a subsequence we could assume that $\beta_{n}$ fixed a point $x_{n}$ in $H_{2}(\infty)$ for every integer $n$. Let $p$ be the unique fixed point of $\beta$ in $H$. By passing to a further subsequence we may assume that $\left\{x_{n}\right\}$ converges to a point $x \in H(\infty)$.

Let $\gamma, \gamma_{n}$ denote, respectively, the geodesics $\gamma_{p x}, \gamma_{p x_{n}}$. The function $\left(d_{\beta_{n}} \circ \gamma_{n}\right)^{2}(t)$ $=d^{2}\left(\gamma_{n} t, \beta_{n} \gamma_{n} t\right)$ is a $C^{\infty}$ convex function on $\mathbf{R}$ which is bounded above for $t \geqslant 0$ since $\beta_{n}$ fixes $x_{n}$. Hence $\left(d_{\beta_{n}} \circ \gamma_{n}\right)(t)$ is nonincreasing in $t$, and it follows that

$$
d_{\beta}(\gamma t)=\lim _{n \rightarrow \infty} d_{\beta_{n}}\left(\gamma_{n} t\right) \leqslant \lim _{n \rightarrow \infty} d_{\beta_{n}}\left(\gamma_{n} 0\right)=\lim _{n \rightarrow \infty} d_{\beta_{n}}(p)=d_{\beta}(p)=0
$$

for every $t \geqslant 0$. Therefore, $\beta$ fixes $\gamma_{p x} t$ for every $t \geqslant 0$, contradicting the fact that $\beta$ has a unique fixed point in $H$. It follows that $\beta_{n}$ has no fixed point in $H(\infty)$ for sufficiently large $n$ and hence is elliptic.

We prove part (3). Let $\Gamma^{*}$ be the subgroup of $\Gamma$ of index $\leqslant 2$ that leaves invariant the foliations of $H$ induced by the tangent spaces of $H_{1}$ and $H_{2}$. Let $M^{*}=H / \Gamma^{*}$ and let $[\alpha]=T(\phi)$ be a free homotopy class of $M^{*}$. In this case we may write $\phi=\phi_{1} \times \phi_{2}$, where $\phi_{i} \in I\left(H_{i}\right), i=1,2$. If either component $\phi_{i}$ is elliptic, then $[\alpha]^{n}$ is bounded for every integer $n \geqslant 1$ by the argument used in part (2). Suppose now that neither component $\phi_{i}$ is elliptic. Let $\gamma(t)=\left(\gamma_{1}(t), \gamma_{2}(t)\right)$ be a geodesic translated by $\phi$ with period $\omega$. Then $\left(\phi_{i} \circ \gamma_{i}\right)(t)=\gamma_{i}(t+\omega)$ for $i=1,2$ and all $t$. Since neither $\phi_{i}$ is elliptic it follows that $\left\|\gamma_{i}^{\prime}\right\|>0$ for $i=1,2$ and, hence, both $\phi_{1}$ and $\phi_{2}$ are hyperbolic. For each integer $k$ define $\gamma_{k}(t)=\left(\gamma_{1}(t), \gamma_{2}(t+k)\right)$. Then $\phi$ translates each geodesic $\gamma_{k}$ with period $\omega$ and clearly $\operatorname{Hd}\left(\gamma, \gamma_{k}\right) \rightarrow+\infty$ as $k \rightarrow+\infty$. Hence $[\alpha]=T(\phi)$ is unbounded.

We showed in (2) that there exist free homotopy classes $[\alpha]$ in $M^{*}$ such that $[\alpha]^{n}$ is bounded for every integer $n \geqslant 1$. It remains only to show that there exist unbounded free homotopy classes $[\alpha]$ in $M^{*}$. By the discussion above we need only demonstrate that there exist elements $\phi=\phi_{1} \times \phi_{2} \in \Gamma^{*}$ such that $\phi_{1}$ and $\phi_{2}$ are both hyperbolic.

Let $\gamma=\left(\gamma_{1}, \gamma_{2}\right)$ be a geodesic of $H$ with $\left\|\gamma_{i}^{\prime}\right\|>0$ for $i=1,2$. Since $H / \Gamma^{*}$ is compact $\Gamma^{*}$ satisfies the duality condition and we can find a sequence $\left\{\phi_{n}\right\} \subseteq \Gamma^{*}$ such that $\phi_{n}(p) \rightarrow \gamma(\infty)$ and $\phi_{n}^{-1}(p) \rightarrow \gamma(-\infty)$ for any point $p \in H[5$, p. 78]. Fix $p=\left(p_{1}, p_{2}\right) \in H$ and write $\phi_{n}=\alpha_{n} \times \beta_{n}$, where $\alpha_{n} \in I\left(H_{1}\right)$ and $\beta_{n} \in I\left(H_{2}\right)$. It is clear that $\alpha_{n}\left(p_{1}\right) \rightarrow \gamma_{1}(\infty), \alpha_{n}^{-1}\left(p_{1}\right) \rightarrow \gamma_{1}(-\infty), \beta_{n}\left(p_{2}\right) \rightarrow \gamma_{2}(\infty)$ and $\beta_{n}^{-1}\left(p_{2}\right) \rightarrow$ $\gamma_{2}(-\infty)$ as $n \rightarrow+\infty$. Since the hyperbolic plane satisfies the Visibility axiom we may apply Propositions 8.4 and 8.6 of [11] separately to the sequences $\left\{\alpha_{n}\right\}$ and $\left\{\beta_{n}\right\}$ to conclude that both $\alpha_{n}$ and $\beta_{n}$ are hyperbolic for sufficiently large $n$. Hence $T\left(\phi_{n}\right)$ is an unbounded free homotopy class in $M^{*}$ for sufficiently large $n$. We remark that although Propositions 8.4 and 8.6 of [11] are stated for discrete isometry groups one needs only slight modifications to make the results apply also to nondiscrete groups such as $\Gamma_{i}^{*}, i=1,2$. This completes the proof of Proposition 3.9 .

Appendix III. We prove the supporting lemmas for Theorem A.

Proof of Lemma A1. Choose a constant $c>0$ such that

$$
\operatorname{Hd}\left(F \circ \gamma[0, \omega], \gamma^{*}\left[0, \omega^{*}\right]\right) \leqslant c .
$$


Then since $\phi^{*}(F \gamma t)=F(\phi \gamma t)=(F \circ \gamma)(t+\omega)$ and $\phi^{*} \gamma^{*} t=\gamma^{*}\left(t+\omega^{*}\right)$ for all $t$, it follows that $\operatorname{Hd}\left(F \circ \gamma(\mathbf{R}), \gamma^{*}(\mathbf{R})\right) \leqslant c$.

Proof of Lemma A2. Let $\sigma$ be a geodesic equivalent to $\gamma$ with $\operatorname{Hd}(\gamma, \sigma)=R>$ $r$. Then for any $t \geqslant \mathbf{R}$ we have

$$
d\left(F(\sigma t), \gamma^{*}\right) \geqslant d(F(\sigma t), F \circ \gamma)-\operatorname{Hd}\left(F \circ \gamma, \gamma^{*}\right) \geqslant d(F(\sigma t), F \circ \gamma)-c .
$$

Given $\varepsilon>0$ and $t \in \mathbf{R}$ choose $t^{*} \in \mathbf{R}$ so that

$$
d(F(\sigma t), F \circ \gamma) \geqslant d\left(F(\sigma t), F\left(\gamma t^{*}\right)\right)-\varepsilon .
$$

Then

$$
d\left(F(\sigma t), F\left(\gamma t^{*}\right)\right) \geqslant \zeta d\left(\sigma t, \gamma t^{*}\right) \geqslant \zeta d(\sigma t, \gamma)=\zeta \operatorname{Hd}(\sigma, \gamma)
$$

since $\operatorname{Hd}(\sigma, \gamma)=R \geqslant r$. The result follows since $\varepsilon>0$ is arbitrary.

Proof of Lemma A3. (1) Since $\sigma$ is equivalent to $\gamma$ it follows that $\sigma_{n}=\phi^{n} \circ \sigma$ is equivalent to $\phi^{n} \circ \gamma=\gamma$ for every positive integer $n$; recall that $\phi$ translates $\gamma$. Moreover, $\operatorname{Hd}\left(\sigma_{n}, \gamma\right)=\operatorname{Hd}(\sigma, \gamma)=R>0$ for every $n$. Let $v_{n} \in T_{\gamma(0)} H$ be the initial velocity of the unit speed geodesic from $\gamma(0)$ to $\sigma_{n}$ that is mutually perpendicular to $\gamma$ and $\sigma_{n}$. Passing to a subsequence $\left\{v_{m_{k}}\right\}$ we may assume that the unit vectors $\left\{v_{m_{k}}\right\}$ converge to a unit vector $v$ in $T_{\gamma(0)} H$. If $p_{m_{k}}=\exp _{\gamma(0)}\left(R v_{m_{k}}\right)$ and $p=\exp _{\gamma(0)}(R v)$, then $p_{m_{k}} \rightarrow p$ as $k \rightarrow+\infty$ and $p_{m_{k}}$ lies in $\sigma_{m_{k}}$ for every $k$. Given $\varepsilon>0$ we can choose an integer $k_{0}>0$ such that if $k \geqslant k_{0}, j \geqslant k_{0}$ then $d\left(p_{m_{k}}, p_{m_{j}}\right)$ $<\varepsilon$. In particular,

$$
\operatorname{Hd}\left(\sigma, \phi^{\left(m_{k}-m_{j}\right)} \circ \sigma\right)=\operatorname{Hd}\left(\sigma_{m_{k}}, \sigma_{m_{j}}\right)=d\left(p_{m_{k}}, \sigma_{m_{j}}\right)<d\left(p_{m_{k}}, p_{m_{j}}\right)<\varepsilon
$$

for $k, j \geqslant k_{0}$.

We have shown that for every $\varepsilon>0$ we can find an arbitrarily large integer $n$ such that $\operatorname{Hd}\left(\sigma, \phi^{n} \circ \sigma\right)<\varepsilon$. The assertion of part (1) of Lemma A3 now follows immediately.

(2) Let $P: H \rightarrow \gamma$ be orthogonal projection and recall that $d(P p, P q) \leqslant d(p, q)$ for all $p, q$ in $H$ by Lemma 3.2 of [2]. Now choose integers $n_{k} \rightarrow+\infty$ as in (1) so that $\operatorname{Hd}\left(\sigma, \phi^{n_{k}} \circ \sigma\right) \rightarrow 0$ as $k \rightarrow+\infty$. Parametrize $\sigma$ so that $P(\sigma 0)=\gamma(0)$.

We assert first that $d\left(\phi^{n_{k}}(\sigma 0), \sigma\left(n_{k} \omega\right)\right) \rightarrow 0$ as $k \rightarrow+\infty$. Let $\beta_{k}$ be that number such that $d\left(\phi^{n_{k}}(\sigma 0), \sigma\left(\beta_{k}\right)\right)=\operatorname{Hd}\left(\phi^{n_{k}} \circ \sigma, \sigma\right)$. Now

$$
P\left(\phi^{n_{k}}(\sigma 0)\right)=\phi^{n_{k}}(P \sigma 0)=\phi^{n_{k}} \gamma(0)=\gamma\left(n_{k} \omega\right)
$$

and $P\left(\sigma \beta_{k}\right)=\gamma\left(\beta_{k}\right)$ for every $k$ since $P(\sigma 0)=\gamma(0)$, which implies $d(\sigma t, \gamma t)=$ $\operatorname{Hd}(\sigma, \gamma)$ and $P(\sigma t)=\gamma(t)$ for every $t \in \mathbf{R}$. Hence

$$
\begin{aligned}
\left|\beta_{k}-n_{k} \omega\right| & =d\left(P\left(\phi^{n_{k}} \sigma 0\right), P\left(\sigma \beta_{k}\right)\right) \leqslant d\left(\phi^{n_{k}} \sigma 0, \sigma \beta_{k}\right) \\
& =\operatorname{Hd}\left(\phi^{n_{k}} \circ \sigma, \sigma\right) \rightarrow 0
\end{aligned}
$$

as $k \rightarrow+\infty$. Finally

$$
\begin{aligned}
d\left(\phi^{n_{k}}(\sigma 0), \sigma\left(n_{k} \omega\right)\right) & \leqslant d\left(\phi^{n_{k}}(\sigma 0), \sigma \beta_{k}\right)+d\left(\sigma \beta_{k}, \sigma n_{k} \omega\right) \\
& =\operatorname{Hd}\left(\phi^{n_{k}} \circ \sigma, \sigma\right)+\left|\beta_{k}-n_{k} \omega\right| \rightarrow 0
\end{aligned}
$$

as $k \rightarrow+\infty$.

The fact that $d\left(\phi^{n_{k}}(\sigma 0), \sigma\left(n_{k} \omega\right)\right) \rightarrow 0$ implies that

$$
d\left(\left(\phi^{*}\right)^{n_{k}}(F \sigma 0), F\left(\sigma n_{k} \omega\right)\right)=d\left(F\left(\phi^{n_{k} \sigma 0}\right), F\left(\sigma n_{k} \omega\right)\right) \rightarrow 0
$$


by the uniform continuity of $F$. If $P^{*}: H^{*} \rightarrow \gamma^{*}$ denotes orthogonal projection, then

$$
\begin{aligned}
\left|s_{k}-n_{k} \omega^{*}\right| & =d\left(\gamma^{*} s_{k}, \gamma^{*} n_{k} \omega^{*}\right)=d\left(P^{*}\left(F \sigma n_{k} \omega\right), P^{*}\left(\phi^{* n_{k}} F \sigma 0\right)\right) \\
& \leqslant d\left(F \sigma n_{k} \omega, \phi^{* n_{k}} F \sigma 0\right) \rightarrow 0
\end{aligned}
$$

as $k \rightarrow+\infty$.

Proof of Lemma A4. We shall need the following

Sublemma. Let $\gamma, \sigma, \beta$ be maximal unit speed geodesics in a Hadamard manifold $H$ such that $\gamma$ and $\sigma$ are orthogonal to $\beta$ at $\beta(0)$ and $\beta(c)$, respectively, where $c>0$. If $\gamma(\infty)=\sigma(\infty)$ then $\gamma[0, \infty), \sigma[0, \infty)$ and $\beta[0, c]$ bound a flat half-strip in $H$.

Proof of The Sublemma. By a flat half-strip we mean a totally geodesic isometric imbedding $F:[0, \infty) \times[0, c] \rightarrow H$ such that $F(t, 0)=\gamma(t), F(t, c)=\sigma(t)$ and $F(0, s)=\beta(s)$ for all $(t, s) \in[0, \infty) \times[0, c]$. Begin by parametrizing $\gamma$ and $\sigma$ so that $\gamma(0)=\beta(0)$ and $\sigma(0)=\beta(c)$. The function $f(t)=d^{2}(\gamma t, \sigma)$ is a $C^{\infty}$ convex function on $\mathbf{R}$ by Theorem 4.1 of [2] and has a critical point, hence a global minimum, at $t=0$ since $\beta$ is a mutual perpendicular to $\gamma$ and $\sigma$. By hypothesis $f$ is bounded above on $[0, \infty)$ since $\gamma(\infty)=\sigma(\infty)$, and hence $f$ is nonincreasing on $\mathbf{R}$. It follows that $f(t) \equiv f(0)$ for $t \geqslant 0$ since $f$ has a global minimum at $t=0$. It now follows by Proposition 5.1 of [11] that $\gamma, \sigma$ and $\beta$ bound a flat half-strip in $H$.

We begin the proof of Lemma A4 by determining numbers $A, R>0$ such that there exists no flat totally geodesic rectangle with height $R$ whose base has length $A$ and lies on $\beta$. We shall then set $A^{*}=A$ and show the existence of a constant $R^{*}>0$ satisfying the hypotheses of Lemma A4. We let $R_{0}=\sup \{r>0$ : there exists a flat strip of width $r$ with $\beta$ as one of the boundary geodesics $\}$. Note that $R_{0}$ is finite since $\beta$ bounds no flat half-planes in $H$. Now fix $R>R_{0}$ arbitrarily.

To determine $A$ we define $A_{0}=\sup \{a>0$ : there exists a flat, totally geodesic rectangle of height $R$ and base length $a$, where the base of the rectangle lies on $\beta$. We assert that $A_{0}$ is finite and then fix $A>A_{0}$ arbitrarily. If $A_{0}$ were not finite then we could find a sequence $\left\{T_{n}\right\}$ of flat, totally geodesic rectangles of height $R$ whose bases lie on $\beta$ and have endpoints $\beta\left(s_{n}\right), \beta\left(t_{n}\right)$, where $0<t_{n}-s_{n} \rightarrow+\infty$ as $n \rightarrow+\infty$. Replacing $T_{n}$ by $\phi^{m_{n}}\left(T_{n}\right)$ for a suitable integer $m_{n}$ we may assume that $s_{n} \rightarrow-\infty$ and $t_{n} \rightarrow+\infty$ as $n \rightarrow+\infty$. Hence some subsequence of the rectangles $\left\{T_{n}\right\}$ converges to a flat strip $T$ of width $R$ with $\beta$ as one of the bounding goedesics, contradicting the choice of $R_{0}$. Therefore $A_{0}$ is finite.

We now set $A^{*}=A$ and first prove Lemma A4 in the special case that $d(P \sigma a, P \sigma b)=A^{*}$. Without loss of generality we may assume that $[a, b]=[0,1]$. Suppose that the assertion of Lemma A4 is false in the special case above for some constant $K>1$. Then we can find a sequence of curves $\sigma_{k}:[0,1] \rightarrow H$ such that $d\left(\sigma_{k}, \beta\right) \rightarrow+\infty, d\left(P \sigma_{k} 0, P \sigma_{k} 1\right)=A^{*}$ and $L\left(\sigma_{k}\right) \leqslant K A^{*}$ for every $k$. Let $p_{k}=\sigma_{k}(0)$ and $q_{k}=\sigma_{k}(1)$. Let $v_{k}, w_{k}$ be unit vectors orthogonal to $\beta^{\prime}\left(c_{k}\right)$ and $\beta^{\prime}\left(c_{k}+A^{*}\right)$ such that $\exp \left(s_{k} v_{k}\right)=p_{k}$ and $\exp \left(t_{k} w_{k}\right)=q_{k}$, where $s_{k}=d\left(p_{k}, \beta\right)$ and $t_{k}=\left(q_{k}, \beta\right)$. Note that $s_{k} \rightarrow+\infty$ and $t_{k} \rightarrow+\infty$ by the hypothesis that $d\left(\sigma_{k}, \beta\right) \rightarrow+\infty$. We may also assume that $0 \leqslant c_{k}<\omega$ for every $k$, where $\omega>0$ is the period of $\phi$, by replacing $\sigma_{k}$ with $\phi^{m_{k}} \circ \sigma_{k}$ for a suitable integer $m_{k}$. 
Passing to a subsequence we may assume that $c_{k} \rightarrow c \in[0, \omega], v_{k} \rightarrow v \in T_{\beta(c)} H$ and $w_{k} \rightarrow w \in T_{\beta\left(c+A^{*}\right)} H$. By continuity $v$ and $w$ are unit vectors orthogonal to $\beta^{\prime}(c)$ and $\beta^{\prime}\left(c+A^{*}\right)$. Passing to a further subsequence we let the sequences $\left\{p_{k}\right\},\left\{q_{k}\right\}$ converge to points $x, y$ in $H(\infty)$. It follows that $x=y$ since $d\left(p_{k}, q_{k}\right) \leqslant$ $L\left(\sigma_{k}\right) \leqslant K A^{*}$ for every $k$. Moreover,

$$
\gamma_{v}(\infty)=\lim _{k \rightarrow \infty} \gamma_{v_{k}}\left(s_{k}\right)=\lim _{k \rightarrow \infty} p_{k}=x
$$

and

$$
\gamma_{w}(\infty)=\lim _{k \rightarrow \infty} \gamma_{w_{k}}\left(t_{k}\right)=\lim _{k \rightarrow \infty} q_{k}=x .
$$

By the sublemma above the geodesics $\gamma_{v}[0, \infty), \gamma_{w}[0, \infty)$ and $\beta\left[c, c+A^{*}\right]$ bound a flat half-strip of width $A^{*}=A$, and this flat half-strip contains a flat totally geodesic rectangle of height $R$ and base $\beta\left[c, c+A^{*}\right]$ of width $A^{*}$, contradicting the choice of $A$ and $R$. Therefore Lemma A4 is proved in the special case that $d(P \sigma a, P \sigma b)=A^{*}$.

Finally we consider the general case that $d(P \sigma a, P \sigma b) \geqslant A^{*}$. Let $K>1$ be given and choose a number $R^{*}>0$ such that if $\sigma:[a, b] \rightarrow H$ is a continuous, piecewise $C^{\infty}$ curve with $d(\sigma, \beta)>R^{*}$ and $d(P \sigma a, P \sigma b)=A^{*}$, then $L(\sigma)>2 K A^{*}$. We assert that $R^{*}>0$ is the desired constant. Let $\sigma:[a, b] \rightarrow H$ be a continuous, piecewise $C^{\infty}$ curve with $d(\sigma, \beta)>R^{*}$ and $d(P \sigma a, P \sigma b)>A^{*}$. We may assume that $P \sigma a=$ $\beta(0)$. Choose an integer $N \geqslant 1$ so that $N A^{*} \leqslant d(P \sigma a, P o b)<(N+1) A^{*}$. Define a partition $a=t_{0}<t_{1}<t_{2}<\cdots<t_{N+1}=b$ so that $P\left(\sigma t_{i}\right)=\beta\left(i A^{*}\right)$ for $1 \leqslant i \leqslant$ $N$. Let $\sigma_{i}$ denote the restriction of $\sigma$ to $\left[t_{i}, t_{i+1}\right]$. By hypothesis $L\left(\sigma_{i}\right)>2 K A^{*}$ for $0 \leqslant i \leqslant N-1$. Note that

$$
L\left(\sigma_{N}\right) \geqslant d\left(\sigma t_{N}, \sigma b\right) \geqslant d\left(P \sigma t_{N}, P \sigma b\right)=B, \quad B=d(P \sigma a, P \sigma b)-N A^{*} .
$$

Note also that $0 \leqslant B<A^{*}$. Hence

$$
L(\sigma)=\sum_{i=0}^{N} L\left(\sigma_{i}\right) \geqslant 2 K N A^{*}+B .
$$

Finally $K N A^{*} \geqslant K A^{*}>(K-1) A^{*}>(K-1) B$, and from this it follows that

$$
2 K N A^{*}+B>K N A^{*}+K B=K\left(N A^{*}+B\right)=K d(P \sigma a, P \sigma b) .
$$

We conclude that $L(\sigma)>K d(P \sigma a, P \sigma b)$, which proves Lemma A4.

\section{BIBLIOGRAPHY}

1. W. Ballmann, Dissertation, University of Bonn, 1978, and Bonner Math. Schriften, vol. 113, 1978.

2. R. Bishop and B. O'Neill, Manifolds of negative curvature, Trans. Amer. Math. Soc. 145 (1969), $1-49$.

3. A. Borel, Compact Clifford-Klein forms of symmetric spaces, Topology 2 (1963), 111-122.

4. S. Chen, Weak rigidity of compact negatively curved manifolds, Pacific J. Math. 78 (1978), 273-278.

5. S. Chen and P. Eberlein, Isometry groups of simply connected manifolds of nonpositive curvature, Illinois J. Math. 24 (1980), 73-103.

6. P. Eberlein, A canonical form for compact nonpositively curved manifolds whose fundamental groups have nontrivial center (submitted).

7. __ Geodesic flow in certain manifolds without conjugate points, Trans. Amer. Math. Soc. 167 (1972), 151-170.

8. Geodesic rigidity in compact nonpositively curved manifolds. II (in preparation).

9. ___ Lattices in spaces of nonpositive curvature, Ann. of Math. (2) 111 (1980), 435-476. 
10. __ Surfaces of nonpositive curvature, Mem. Amer. Math. Soc. No. 218 (1979).

11. P. Eberlein and B. O'Neill, Visibility manifolds, Pacific J. Math. 46 (1973), 45-109.

12. W. Fenchel and J. Nielsen, Discontinuous groups of non-Euclidean motions (to appear).

13. D. Gromoll and J. Wolf, Some relations between the metric structure and the algebraic structure of the fundamental group in manifolds of nompositive curvature, Bull. Amer. Math. Soc. 77 (1971), 545-552.

14. M. Gromov, Three remarks on geodesic dynamic and fundamental group (preprint).

15. __ Manifolds of negative curvature, J. Differential Geom. 13 (1978), 223-230.

16. E. Heintze, Mannigfaltigkeiten negativer Krummung, Habilitationschrift, University of Bonn, 1976.

17. W. Klingenberg, Geodatischer Fluss auf Mannigfaltigkeiten vom hyperbolischen Typ, Invent. Math. 14 (1971), 63-82.

18. S. Kobayashi and K. Nomizu, Foundations of differential geometry, Vol. 1, Wiley, New York, 1963, pp. 179-193.

19. H. B. Lawson and S.-T. Yau, Compact manifolds of nonpositive curvature, J. Differential Geom. 7 (1972), 211-228.

20. A. Marden, Isomorphisms between Fuchsian groups, Lecture Notes in Math., vol. 505, SpringerVerlag, Berlin and New York, 1978, pp. 56-78.

21. M. Morse, A fundamental class of geodesics on any closed surface of gemus greater than one, Trans. Amer. Math. Soc. 26 (1924), 25-60.

22. G. D. Mostow, Strong rigidity of locally symmetric spaces, Ann. of Math Studies, No. 78, Princeton Univ. Press, Princeton, N. J., 1973.

23. M. S. Raghunathan, Discrete subgroups of Lie groups, Springer-Verlag, New York, 1972.

24. H. Shimizu, On discontinuous groups operating on the product of upper half planes, Ann. of Math. (2) 77 (1963), 33-71.

25. E. Spanier, Algebraic topology, McGraw-Hill, New York, 1966.

26. N. Steenrod, The topology of fiber bundles, Princeton Univ. Press, Princeton, N. J., 1951.

27. J. Wolf, Homogeneity and bounded isometries in manifolds of negative curvature, Illinois J. Math. 8 (1964), 14-18.

28. Spaces of constant curvature, 2nd ed., published by the author, Berkeley, Calif., 1972.

29. S.-T. Yau, On the fundamental group of compact manifolds of nonpositive curvature, Ann. of Math. (2) 93 (1971), 579-585.

Department of Mathematics, University of North Carolina, Chapel Hill, North Carolina 27514 